\title{
Baltic provenance of top-Famennian siliciclastic material of the northern Rhenish Massif, Rhenohercynian zone of the Variscan orogen
}

\author{
Katarzyna Kołtonik ${ }^{1,2} \cdot$ Agnieszka Pisarzowska $^{1} \cdot$ Mariusz Paszkowski $^{1} \cdot$ Jiří Sláma $^{3} \cdot$ Ralph Thomas Becker $^{4}$. \\ Marek Szczerba ${ }^{1} \cdot$ Wojciech Krawczyński ${ }^{2}$. Sven Hartenfels ${ }^{4} \cdot$ Leszek Marynowski $^{2}$
}

Received: 12 July 2017 / Accepted: 11 June 2018 / Published online: 9 July 2018

(C) The Author(s) 2018

\begin{abstract}
The provenance of top-Famennian sedimentary rocks linked to the Hangenberg Crisis from the northern Rhenish Massif (Germany) was investigated by the means of detrital zircon U-Pb geochronology. Based on the obtained age spectra, three main tectonothermal domains are recognized as possible sources: Paleo- and Mesoproterozoic ( 2000-1000 Ma) units of Baltica and Early Paleozoic Caledonian orogen ( 500-400 Ma). Our interpretation of the detritus having been derived from northern source areas, i.e., Baltica and the Scandinavian Caledonides, with a minor input of German-Polish (RügenPomeranian) Caledonides, contradicts the traditional view that, during the Upper Devonian, the northern Rhenish Massif was supplied by detritus from the south. Complementary mineralogical, textural and geochemical analyses point to a derivation of the detritus of Drewer and Hangenberg Sandstones mainly from felsic, recycled continental crust. The elevated concentrations of $\mathrm{Pb}$ and $\mathrm{Zn}$ in the studied sections are a feature attributed to hydrothermal alteration related to the terminal Devonian synsedimentary volcanism or post-depositional Variscan deformation.
\end{abstract}

Keywords Detrital zircon $\cdot$ Famennian sandstones $\cdot$ Hangenberg event $\cdot$ Baltica

\section{Introduction}

The Rhenish Massif in western Germany with its complex Paleozoic evolution represents a piece of puzzle in the understanding of the processes that led to the polyphase

Electronic supplementary material The online version of this article (https://doi.org/10.1007/s00531-018-1628-4) contains supplementary material, which is available to authorized users.

Katarzyna Kołtonik

ndkolton@cyf-kr.edu.pl

1 Institute of Geological Sciences, Polish Academy of Sciences, Kraków Research Centre, Senacka 1, 31002 Kraków, Poland

2 Faculty of Earth Sciences, University of Silesia, Będzińska 60, 41200 Sosnowiec, Poland

3 Institute of Geology, Czech Academy of Sciences, Rozvojová 269, 16500 Praha, Czech Republic

4 Institut für Geologie und Paläontologie, Westfälische Wilhelms-Universität, Corrensstrasse 24, 481499 Münster, Germany collision of Gondwana and Laurussia terranes and the Devonian-Carboniferous Variscan orogeny (see review by Franke et al. 2017). During the Upper Devonian, the area of the recent Rhenish Massif represented a part of the Rhenohercynian Basin, where sedimentation was controlled by sea level fluctuations, synsedimentary tectonic activity, and a changing supply of siliciclastic material (e.g., Paproth 1986; Königshof et al. 2016). Sedimentological, paleogeographic, and petrological analyses of Upper Devonian sedimentary rocks of the northern Rhenish Massif (e.g., Paproth 1986; Bless et al. 1993) indicated their derivation from southern sources (adjoining highs/shoals). However, Schulz-Dobrick and Wedepohl (1983), Press (1986), and Haverkamp (1991) suggested that the main detritus was shed from the north ("Old Red Continent") into the Rhenohercynian Basin during the Lower and Middle Devonian. Later studies indicated that the Silurian and Devonian siliciclastic material of the neighbouring Mid-German Crystalline Rise and Harz Mountains was supplied from northern sources, e.g., Baltica as a part of the Laurussia paleocontinent (e.g., Geisler et al. 2005; Zeh and Gerdes 2010). However, in a recent paper, Dörr et al. (2017) excluded a Baltic source for detrital 
zircons from Upper Devonian clastic (meta)sediments of the Böllstein Odenwald (Mid-German-Crystalline-Zone). A potential source area for Upper Devonian detritus in the Rhenohercynian Basin could also have been shoals known from the Rügen area (northestern Germany; e.g., McCann 1999). The provenance of Upper Devonian sedimentary rocks of the northern part of the Rhenish Massif has not been studied in detail before. Detrital zircon provenance data exist for Devonian and Carboniferous sedimentary rocks of the southeastern and eastern part of the Rhenish massif though (e.g., Haverkamp et al. 1992; Eckelmann et al. 2014; Franke and Dulce 2017) and can be used for detailed correlations.

Provenance analysis based on U-Pb dating of detrital zircon is nowadays a routine method for resolving paleogeographic and plate tectonic settings. Supplemented with mineralogical and geochemical data, it may provide insights into the geodynamic processes at different time scales. The main aim of this paper is to define the source areas of uppermost Fammenian siliciclastic rocks (sandstone, siltstone and mudstone; see below for explanation) from the northern Rhenish Massif based on U-Pb dating of detrital zircon and mineralogical and geochemical analysis of the sedimentary rocks. These data also allow to test the presence of the GermanPolish (Rügen-Pomeranian) Caledonides as an uplifted land in the Rügen area during the Upper Devonian.

\section{Geological setting}

The Paleozoic Variscan orogen is one of the most significant examples of large-scale collisional tectonics with complicated interactions between different terranes or microplates and several narrow oceans (e.g., Franke and Oncken 1990, 1995; Franke 2000; Franke et al. 2017). The classical subdivision of the Variscan mountain belt in central Europe follows the early work of Kossmat (1927). From northwest to southeast, three successive zones with different metamorphic history were defined: the Rhenohercynian, the Saxothuringian, and the Moldanubian units (Fig. 1a). An additional zone, the strongly metamorphosed Mid-German Crystalline High, situated between the Rhenohercynian and Saxothuringian zones, was defined by Brinkmann (1948).

The Rhenohercynian unit is a typical example of a foldand-thrust belt and is best exposed in the Rhenish Massif (e.g., Oncken et al. 1999; Franke 2000), with continuations westwards into the Ardennes, to southwest England, and to the Harz Mountains in the east. It is generally accepted that the basement of Rhenish Massif belongs to the Avalonia Terrane (e.g., Franke 2000). Separation of Avalonia from Gondwana started with a Late Cambrian rifting episode, followed by Lower Ordovician drift northwards that led to the opening of the Rheic Ocean in the south (e.g., Oncken et al. 2000; Linnemann et al. 2008, 2010; Eckelmann et al. 2014).
The docking of Avalonia to Baltica during the Upper Ordovician to middle Silurian ( 450-430 Ma) resulted in the progressive closure of the oceanic Tornquist Sea. During the upper Silurian $(\sim 420 \mathrm{Ma})$, the Iapetus Ocean finally closed due to the collision of Baltica and Avalonia with Laurentia. As a result, the Laurussia paleocontinent, i.e., Old Red Continent (Linnemann et al. 2008; Nance et al. 2010), was formed. Incipient stages of the Variscan orogeny were connected with the closure of the Rheic Ocean by the northward migration of the Armorican Terrane Assemblage (Franke 1995) during the upper Silurian-Lower Devonian (Sánchez Martínez et al. 2007; Nance et al. 2010). This was followed by the opening of the narrow Rhenohercynian Ocean southeast of the Rheic Ocean (Franke et al. 2017). Subductionrelated volcanism lasted into the Lower Carboniferous (e.g., Franke et al. 2017).

\section{The Rhenish Massif}

The Rhenish Massif is composed of an autochthon onto which several allochthonous tectonic units are superimposed (e.g., Franke 1995; Eckelmann et al. 2014; Franke et al. 2017). The oldest deposits known from the northern autochthonous part of the massif are represented by Ordovician and uppermost Silurian sedimentary rocks exposed in the cores of the Ebbe and Remscheid-Altena anticlines (Fig. 1b; Langenstrassen 1983). Infrequent outcrops of pre-Devonian rocks also occur in the southern Rhenish Massif including the Giessen, Marburg and Kellerwald areas (Fig. 1b, e.g., Langenstrassen 1983). The autochthonous Devonian sedimentary sequence is composed of shallow shelf deposits ("Rhenish Magnafacies" and its subfacies, Jansen 2016) and deeper-water, pelagic sedimentary rocks of more distal shelf basins, seamounts, and synsedimentary structural highs ("Hercynian Magnafacies"; e.g., Krebs 1979; Franke 1995; Stets and Schäfer 2002; Becker 2008). The allochthonous units, represented by ocean floor and active margin deposits, are exposed in the southern Rhenish Massif and can be divided into belts known as the Lohra, Steinhorn, Hörre, and Giessen nappes (Franke 1995). A Gondwanan source of detritus for Devonian and Lower Carboniferous allochthonous sedimentary rocks was determined by Huckriede at al. (2004) and Eckelmann et al. (2014) based on muscovite and detrital zircon ages. This suggests a significant late Variscan northwards transport from an original position south of the Rheic Suture and north of the preserved part of the MidGerman Crystalline High (Franke et al. 2017).

During the Early Devonian, the Rhenohercynian Basin was located at the southern margin of Laurussia (e.g., Franke 2000). The southern boundary of the Rhenohercynian Basin was formed by the source regions of the allochthonous units and by the Armorican Terrane Assemblage of peri-Gonwanian origin that later formed the Mid-German 
a
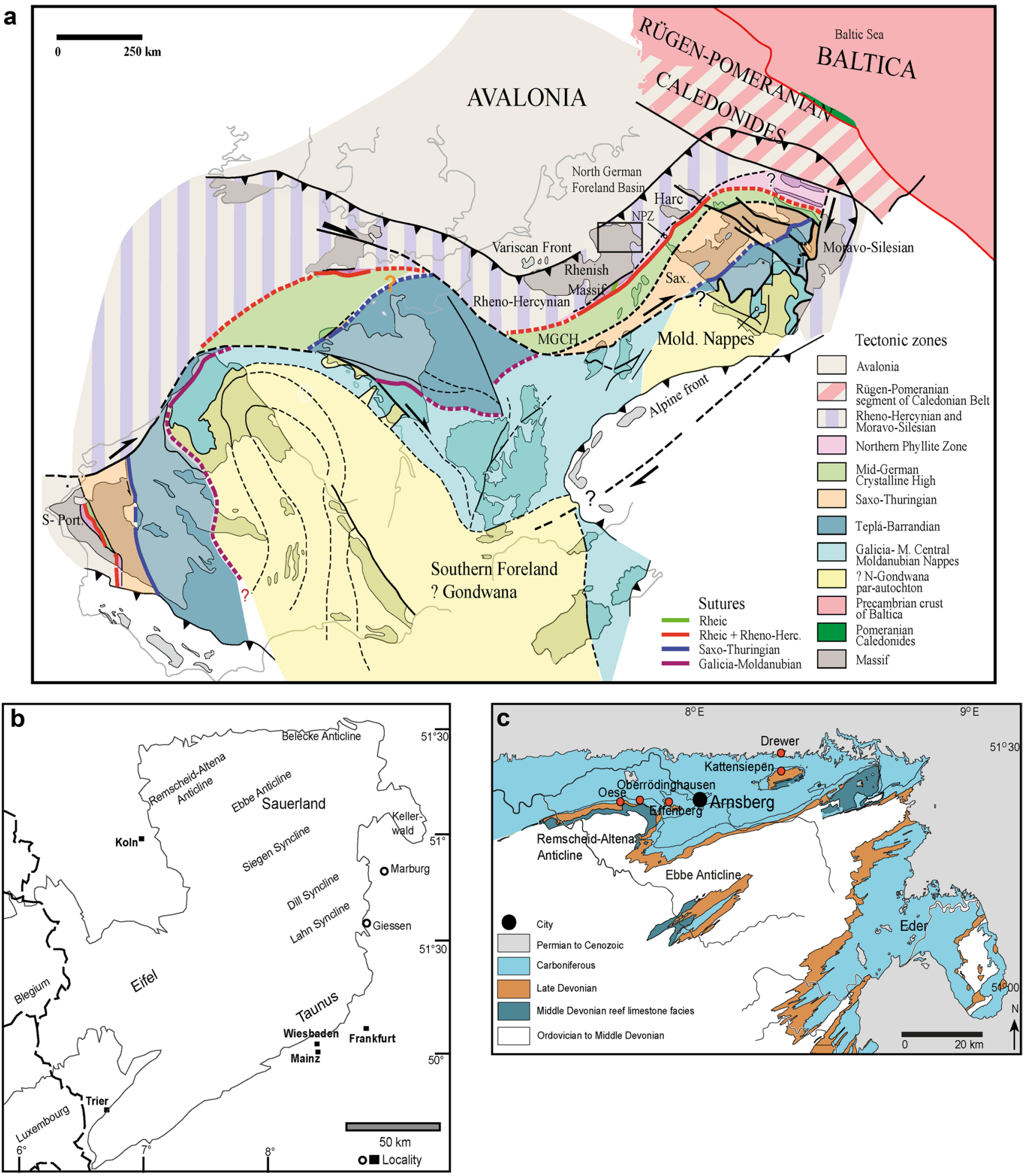

Fig. 1 a Geological overview of the European Variscides (after Grad et al. 2002; Linnemann et al. 2012; Franke 2014) with major tectonic structures. A black rectangle marks the study area shown in c. $N P Z$ northern phyllite zone, $M G C H$ mid-German crystalline high, Mod. Nappes moldanubian nappes, S-Port. south Portugal, Rheno-Herc.

rheno-hercynian, Sax. saxo-thuringian, $M$. massif. b Sketch map of the Rhenish Massif showing the main regions. c Geological map of the northern Rhenish Massif modified from Korn (2004) with marked positions (red points) of the studied sections 
Crystalline High. The siliciclastic material filling of the Rhenohercynian Basin derived mostly from northern sources, i.e., Laurussia (e.g., Langenstrassen 1983; Bełka et al. 2010), but also from southern sources, i.e., from the exposed Silurian magmatic arc related to the Rheic Ocean, from the northern Armorica terranes ("Franconia") that are only partially preserved in the Taunus (the southern Rhenish Massif; Fig. 1b), and also from the Mid-German Crystalline High (Hahn 1990; Hahn and Zankl 1991; Franke et al. 2017). The opening of the narrow Rhenohercynian Basin generated a barrier in the form of a trap for northward transported detritus. The Lower Devonian sedimentary rocks of the Rhenish Massif are mostly sandstone and siltstone (review in Jansen 2016), whereas Middle Devonian rocks are represented by silty sandstone, limestone, and shale (e.g., Langenstrassen 1983; Königshof et al. 2016). The formation of partly large Givetian reef carbonates reflects directly the decrease of detritus influx into the basin (e.g., Franke 2000). This may have been controlled by the change of climate and weathering regime on the Old Red Continent (Becker et al. 2016). Magmatic activity is represented by upper Emsian felsic volcanism in the southern Rhenish Massif (Kirnbauer 1991; Franke 2000), intensive Middle/Upper Devonian (middle Givetian to middle Frasnian) basaltic volcanism recorded in the Lahn-Dill Syncline and Sauerland (Fig. 1b, e.g., Sunkel 1990), and Lower/Middle Devonian (Emsian-Eifelian) keratophyre volcanism recorded in the Siegen and Ebbe anticlines in the central Rhenish Massif (Fig. 1b; Loske and Miller 1987; Winter 2006).

Thin but wide-spread layers of subaerial, explosive volcanic deposits of rhyolitic to trachyandesitic composition characterize the Upper Devonian (Frasnian to lower Famennian) and provide evidence for ongoing subduction of oceanic crust below the Laurussia paleocontinent (Winter 2015). The Upper Devonian sedimentary stack consists of shale, limestone (drowning reef complexed and their talus, followed by condensed, pelagic seamount deposits), and turbiditic sandstone (e.g., Königshof et al. 2016). During Upper Devonian time, eustatic regressions led to episodic sand influxes into the basin (Nehden, Hemberg, Dasberg, Drewer and Hangenberg Sandstones; Krebs 1979; Königshof et al. 2016). It is believed that the Remscheid-Altena shoal that subsequently formed the Variscian Remscheid-Altena Anticline (Fig. 1b) was a sediment source of terrigenous detritus during the Upper Devonian, with fluvial shedding to the north (Keupp and Kompa 1984; Paproth 1986, 1991; Paproth et al. 1986). From the Upper Devonian onward, the Mid-German Crystalline High, which was then the active southern margin of the Rhenohercynian Basin, became a source area for the detrital material in the south (e.g., Franke and Engel 1986; Franke and Dulce 2017). The Lower Carboniferous sedimentary rocks of the northern Rhenish Massif represent passive margin deposits (chert, shale, neritic, pelagic and turbiditic limestone of the "Kohlenkalk" and Culm facies) whilst synorogenic deposition of immature sand of polymictic greywacke composition prograded from the south to north (e.g., Königshof et al. 2016).

\section{Samples}

The Famennian sequence of the northern Rhenish Massif contains five discrete sandstone horizons: the Nehden (= "Plattensandstein"), Hemberg, Dasberg, Drewer, and Hangenberg Sandstone. In this study, we focus on the two youngest units, i.e., the Drewer and Hangenberg Sandstones (Figs. 2, 3).

Sand and silt fractions are crucial as potential source of accessory, detrital minerals grains, opposite to finer fractions, which are very poor in accessory minerals. Sand- and siltstones were identified by both macro- and microscopic observations. The Drewer and Hangenberg Sandstones, Hangenberg Black Shale (HBS) and Hangenberg Shale are established lithostratigraphic units with almost isochronous boundaries (Becker et al. 2016a, b, c), controlled by glacioeustatic changes of sea-level. The lithological inventory of the Drewer and Hangenberg Sandstones in the studied sections covers siliciclastic rocks with different fractions from clay up to gravel (according to the classification scale of Wentworth 1922). In particular sections, silt is the coarsest fraction found (Drewer and Effenberg), while in others, the Hangenberg Sandstone includes true sandstones (e.g., Oese). The HBS and Hangenberg Shale consist mainly of mud and clay fractions. The exception is Effenberg section, where the HBS is represented by mudstone intercalated with thin layers of siltstone up to very fine sandstone. To emphasize the dominant fraction of the studied sedimentary rocks, three simplified types were distinguished: sandstone, siltstone, and mudstone. To avoid stratigraphic confusion, the formal lithological names of the studied units (Drewer and Hangenberg Sandstones, Hangenberg Black Shale and Hangenberg Shale) are used even if the rocks are locally variably siltstone or mudstone.

The Drewer Sandstone is restricted to its type locality of the Belecke Anticline (Figs. 1b, 3) and is a laminated, micarich and pyrite-rich calcareous siltstone with a low-diversity pelagic fauna that pinches out laterally (Schmidt 1924; Korn 1984; Korn et al. 1994; Kumpan et al. 2015; Becker et al. 2016). It accumulated during the topmost Famennian (Upper Devonian VI-D) and marks the onset or regressive prelude of the global Hangenberg Crisis (Kaiser et al. 2015), slightly below the famous Hangenberg Black Shale, which falls in Upper Devonian VI-E.

The Hangenberg Sandstone is composed of sandstone and siltstone and represents a basin-floor fan (Kaiser et al. 2015). It is rich in mica, with infrequent quartzite and chert pebbles 


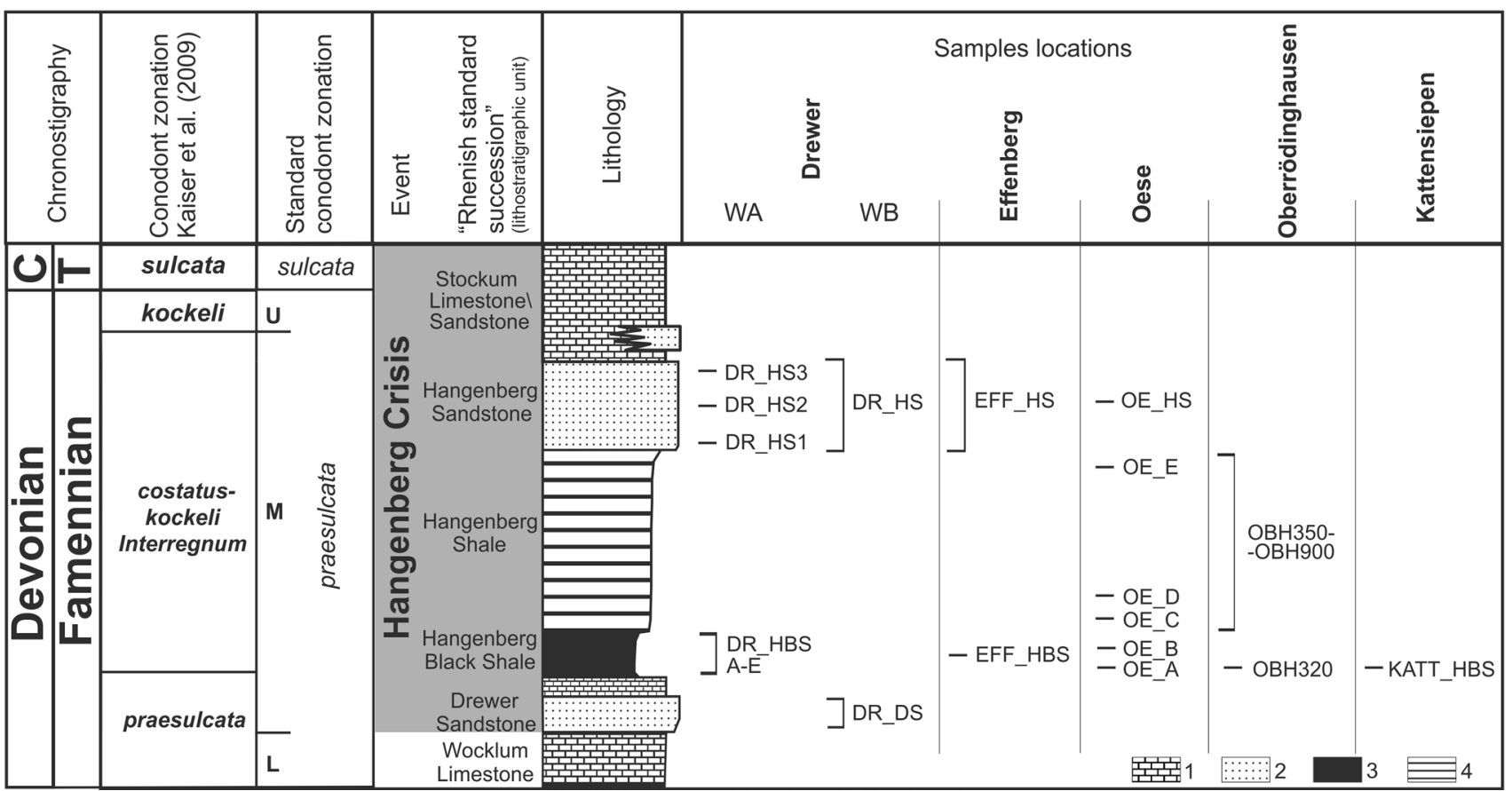

Fig. 2 Generalized stratigraphy of the Devonian/Carboniferous boundary beds in the northern part of the Rhenish Massif with sample positions (Kaiser et al. 2015; Becker et al. 2016a, b, c, modified). WA and WB-sections in Drewer described by Korn (1991).
(1) Limestone, (2) sandstone and siltstone (3) black mudstone, (4) greenish-grey mudstone. $C$ carboniferous, $T$ tournaisian, $L$ lower, $M$ middle, $U$ upper
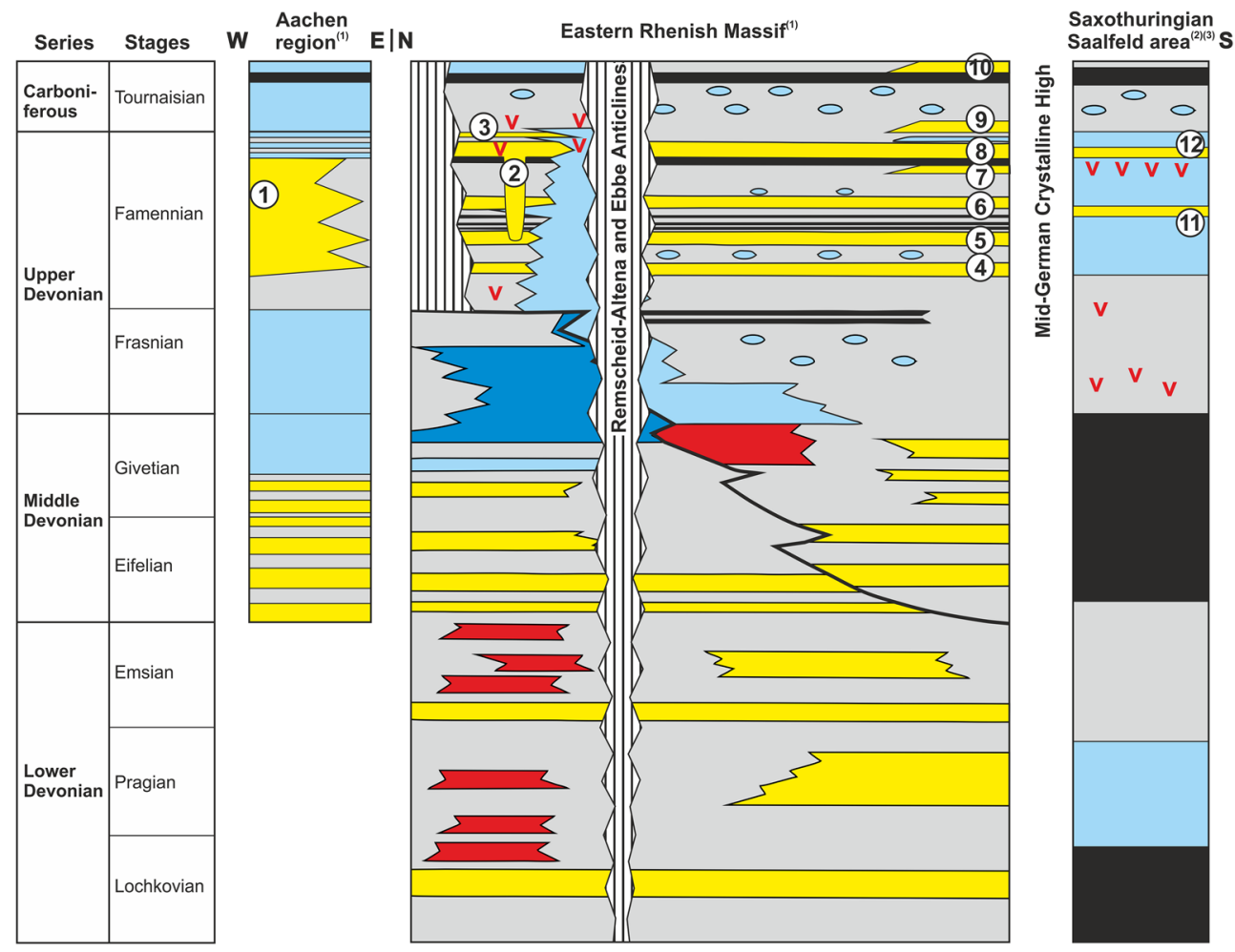

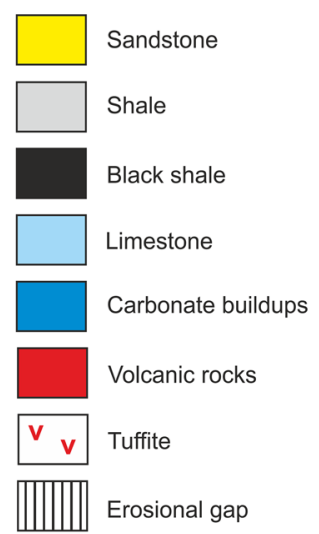

Upper Devonian - Lower Carboniferous siliciclastic formations:

1 - Condroz Group Sandstone

2 - Seiler Conglomerate

3 - Stockum Sandstone

4 - Nehden Sandstone

5 - Hemberg Sandstone

6 - Dasberg Sandstone

7 - Drewer Sandstone

8 - Hangenberg Sandstone

9 - Endbach Sandstone

10 - Kammquartzite

11 - Hauptquarzite

12 - Hangender Quarzite
Fig. 3 Generalized lithostratigraphy of the Rhenish Massif to Saxothuringian Saalfeld area (Southeast German) with stratigraphic position of the Famennian-Tournaisian sandstone units after ${ }^{(1)}$ Bełka et al. (2010), (2) Crônier (2007) and (3) Pfeiffer (1954) and https ://www.thueringen.de/imperia/md/content/tlug/geologie/geoto pe/11_5334_anlage.pdf 
and plant debris (Keupp and Kompa 1984). It represents the regressive peak in the middle Hangenberg Crisis Interval (Kaiser et al. 2015, lower part of Upper Devonian VI-F).

Sandstone and siltstone samples were collected at Drewer, Effenberg, and Oese located in the northern part of the Rhenish Massif (Figs. 1c, 2). The Oese section lies on the northern flank of the Remscheid-Altena Anticline (e.g., Paproth and Streel 1982; Becker et al. 1993), Effenberg on its anticlinal crest (e.g., Luppold et al. 1994; Hartenfels 2011; Hartenfels and Becker 2016), and Drewer represents the Belecke Anticline (Klein 2016; Becker et al. 2016). In the abandoned quarry at Drewer (N51 $\left.{ }^{\circ} 29.616^{\prime} ; \mathrm{E}^{\circ} 21.360^{\prime}\right)$ one siltstone sample of the Drewer Sandstone (DR_DS; Section WB in Korn 1991) and four siltstone samples of Hangenberg Sandstone (samples DR_HS1, DR_HS2 and DR_HS3 from Section WA and sample DR_HS from Section WB in Korn 1991) were collected from its western wall. Sandstone and siltstone samples of the Hangenberg Sandstone were also obtained at Oese (N51 $\left.{ }^{\circ} 24.090^{\prime} ; \mathrm{E}^{\circ} 47.262^{\prime}\right)$ from the small abandoned quarry along the road B7 between Menden and Hemer (sample OE_HS) and from the large, active Effenberg Quarry (N51 ${ }^{\circ} 23.400^{\prime}$; E7 ${ }^{\circ} 57.583^{\prime}$; sample EFF HS).

For comparison, mudstone representing the Hangenberg Black Shale (HBS) and the overlying greenish-grey Hangenberg Shale were collected at Drewer, Oese, Effenberg, Ober-

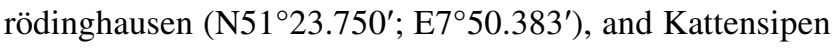
(N51 ${ }^{\circ} 27.950^{\prime}$; E8 $23.616^{\prime}$ ) sections (Fig. 1c).

\section{Methods}

\section{LA-ICP-MS U-Pb zircon dating}

93 to 116 zircon grains with an average grain size of 40 to $120 \mu \mathrm{m}$ from four samples from the Drewer and Hangenberg Sandstones were dated by laser ablation inductively coupled plasma mass spectrometry (LA-ICP-MS). The samples were crushed in a metal mortar and sieved for the fraction $<315 \mu \mathrm{m}$. Magnetic separation (Frantz isodynamic separator) and heavy liquid separation (methylene iodide of $3.32 \mathrm{~g} / \mathrm{cm}^{3}$ density) were used to obtain the zircon concentrate. Zircon grains were hand-picked under a binocular microscope, mounted in epoxy resin, and polished to about half-thickness. Prior to U-Pb analysis, the zircon mounts were imaged with cathodoluminescence (CL) on a scanning electron microscope at the Institute of Petrology and Structural Geology, Charles University in Prague. A Thermo Scientific Element 2 sector field ICP-MS coupled to a $193 \mathrm{~nm}$ ArF excimer laser (Teledyne Cetac Analyte Excite laser) at the Institute of Geology of the Czech Academy of Sciences, Prague, Czech Republic was used to measure the $\mathrm{Pb} / \mathrm{U}$ and $\mathrm{Pb}$ isotopic ratios. The laser was fired at a repetition rate of
$5 \mathrm{~Hz}$ and fluence of $4.51 \mathrm{~J} / \mathrm{cm}^{2}$, with a $20 \mu \mathrm{m}$ spot size. The He carrier gas was flushed through the two-volume ablation cell at a flow rate of $0.7 \mathrm{l} / \mathrm{min}$ and mixed with $0.66 \mathrm{l} / \mathrm{min} \mathrm{Ar}$ and $0.004 \mathrm{l} / \mathrm{min} \mathrm{N}$ prior to introduction into the ICP. The in-house glass signal homogenizer (design of Tunheng and Hirata 2004) was used for mixing all the gases and aerosol resulting in a smooth, spike-free signal. The signal was tuned for maximum sensitivity of $\mathrm{Pb}$ and $\mathrm{U}$, a Th/ $\mathrm{U}$ ratio close to unity and low oxide level, commonly below $0.2 \%$. Typical acquisitions consisted of $15 \mathrm{~s}$ measurement of blank followed by measurements of $\mathrm{U}$, Th and $\mathrm{Pb}$ signals from the ablated zircon for another $35 \mathrm{~s}$. The total of 420 massscans was acquired in the time resolved—peak jumping—pulse counting/analog mode with 1 point measured per peak for masses ${ }^{204} \mathrm{~Pb}+\mathrm{Hg},{ }^{206} \mathrm{~Pb},{ }^{207} \mathrm{~Pb},{ }^{208} \mathrm{~Pb},{ }^{232} \mathrm{Th},{ }^{235} \mathrm{U}$ and ${ }^{238} \mathrm{U}$. Due to a non-linear transition between the counting and analog acquisition modes of the ICP instrument, the raw data were pre-processed using a dedicated Excel macro. As a result, the intensities of ${ }^{238} \mathrm{U}$ were left unchanged if measured in a counting mode and recalculated from ${ }^{235} \mathrm{U}$ intensities if the ${ }^{238} \mathrm{U}$ was acquired in analog mode. Data reduction was then carried out offline using the Iolite data reduction package version 3.4 with VizualAge utility (Petrus and Kamber 2012). Full details of the data reduction methodology can be found in Paton et al. (2010). The data reduction included the correction for gas blank, laser-induced elemental fractionation of $\mathrm{Pb}$ and $\mathrm{U}$, and instrument mass bias. For the data presented here, blank intensities and instrumental bias were interpolated using an automatic spline function while down-hole inter-element fractionation was corrected using an exponential function. No common $\mathrm{Pb}$ correction was applied to the data due to the high $\mathrm{Hg}$ contamination of the commercially available He carrier gas, which precludes accurate correction of the interfering ${ }^{204} \mathrm{Hg}$ on the very small signal of ${ }^{204} \mathrm{~Pb}$ (common lead). Residual elemental fractionation and instrumental mass bias were corrected by normalization to the Plešovice natural zircon reference material (Sláma et al. 2008). Zircon reference material GJ-1 \{no. 63\} (Jackson et al. 2004) and 91500 (Wiedenbeck et al. 1995) were periodically analyzed during the measurements for quality control. The values obtained from analyses performed over many days (discordant GJ-1: mean concordia age of $600 \pm 3 \mathrm{Ma}(2 \sigma)$, mean ${ }^{207} \mathrm{~Pb} /{ }^{206} \mathrm{~Pb}$ age of $608 \pm 2 \mathrm{Ma}(2 \sigma)$; near-concordant 91500: mean concordia age of $1065 \pm 5 \mathrm{Ma}(2 \sigma))$ correspond perfectly and are less than $1 \%$ accurate within the published reference values (GJ$1:{ }^{206} \mathrm{~Pb} /{ }^{238} \mathrm{U}$ age of $600.5 \pm 0.4 \mathrm{Ma}$, Schaltegger et al. 2015 and ${ }^{207} \mathrm{~Pb} /{ }^{206} \mathrm{~Pb}$ age of $608.53 \pm 0.4 \mathrm{Ma}$; Jackson et al. 2004, respectively; 91500: ${ }^{207} \mathrm{~Pb} /{ }^{206} \mathrm{~Pb}$ age of $1065.4 \pm 0.3 \mathrm{Ma}$; Wiedenbeck et al. 1995). The zircon U-Pb ages are presented as probability density plots generated with the ISOPLOT program v. 3.70 (Ludwig 2008). Only analyses showing discordance less than $10 \%$ were taken into account. Of the ages 
determined, 87 of 100 (DR_DS), 94 of 115 (EFF) and 102 of 109 (OE) ages are concordant (exception: DR_HS with 64 of 93 concordant ages). For the data interpretation, the ${ }^{207} \mathrm{~Pb} /{ }^{206} \mathrm{~Pb}$ age was used for zircon older than $1 \mathrm{Ga}$, whereas the ${ }^{206} \mathrm{~Pb} /{ }^{238} \mathrm{U}$ age was used for zircon younger than $1 \mathrm{Ga}$. A summary of the isotope ratio measurements and dating results is presented in Supplement 1. Age errors are quoted at 2 sigma level.

\section{Bulk chemical analysis}

The studied samples were powdered in an agate mill for bulk chemical analysis. The major and trace elements concentrations' measurements were performed on an ICP-MS at Bureau Veritas Mineral Laboratories, Vancouver, Canada. The accuracy and reproducibility of the results were monitored by the repeated analyses of international standard reference materials along the analytical session. Precision of the results was better than $\pm 0.05 \%$ (mostly $\pm 0.01 \%$ ) for the major elements and generally better than $\pm 1 \mathrm{ppm}$ for the trace elements.

The chemical index of alteration (CIA) was calculated using molecular proportions as given in the equation: $\mathrm{CIA}=$ $\left[\mathrm{Al}_{2} \mathrm{O}_{3} /\left(\mathrm{Al}_{2} \mathrm{O}_{3}+\mathrm{CaO} *+\mathrm{Na}_{2} \mathrm{O}+\mathrm{K}_{2} \mathrm{O}\right)\right] \times 100$ (Nesbitt and Young 1982), where $\mathrm{CaO}^{*}$ is the amount of $\mathrm{CaO}$ incorporated in the silicate fraction of the rock. The $\mathrm{CaO}^{*}$ content was calculated based on XRD analyses: carbonate $\mathrm{CaO}$ in ankerite and calcite was subtracted from total $\mathrm{CaO}$. If result of this equation was lower than zero, then the $\mathrm{CaO}^{*}$ content was set to zero. The mineralogical index of alteration was calculated as: MIA = [quartz/(quartz + K-feldspar + plagioclase) $] \times 100$ (Nesbitt et al. 1996, 1997), using proportions of mineral weight percentages derived from XRD analyses.

\section{Bulk rock mineral XRD quantification}

The sample preparation procedure for mineral quantification involved gentle crushing of sample aliquots in a mortar to pass through a $0.4 \mathrm{~mm}$ sieve. An $\mathrm{ZnO}$ internal standard was added in the amount of $10 \%$, followed by McCrone milling with methanol (Środoń et al. 2001).

$\mathrm{X}$-ray diffraction (XRD) analysis was performed using a side-loading procedure to maximize random arrangement of mineral grains. Diffractograms were recorded on a Thermo ARL X'Tra system, CuK $\alpha$ radiation, equipped with a Peltiercooled solid-state detector. The quantitative mineralogy was derived from a whole pattern fitting of experimental diffractograms, with a collection of registered pure standards using an in-house software (Q-Min). During the matching procedure, some parts of diffraction pattern are given higher weighting factors. These are typically ranges, for which stable reflections of internal standard and clay minerals are located.

\section{Results}

\section{Detrital zircon morphology}

Zircon grains have variable shapes and degrees of roundness, from euhedral or slightly abraded to completely rounded grains. There is no visible correlation between the crystallization age of zircon grains and the degree of roundness (Supplement 2). The typical grain size is 40 to $120 \mu \mathrm{m}$, with a majority below $80 \mu \mathrm{m}$ and with most grains characterized by oscillatory zoning (Supplement 1), which is a diagnostic feature of zircon of magmatic origin (Corfu et al. 2003). Some zircon grains reveal a faint or homogenous zonation that may suggest metamorphic growth or modification (Corfu et al. 2003).

\section{Detrital zircon $\mathrm{U}-\mathrm{Pb}$ ages}

The detrital zircon age distributions of all studied Hangenberg and Drewer Sandstone samples are very uniform. The dominant age populations are ca. 2.0 Ga to $900 \mathrm{Ma}$ and 500-400 Ma (Fig. 4). DR_DS also includes a subordinate group at $530-510 \mathrm{Ma}$. A further difference is the presence of an older zircon population of ca. 2.9-2.6 Ga in sample DR_DS (Fig. 4). The Th/U ratios in the dated zircon grains are mostly higher than 0.1 (Supplement 2 ).

\section{Mineralogy and petrography}

The samples from Drewer (DR_HS1, DR_HS2, DR_DS) and Effenberg (EFF_HS) are coarse siltstone with grain sizes of 30-50 $\mu \mathrm{m}$ (Supplement 3). The sample from Oese (OE_HS) represents fine-grained sandstone with grain size 50-300 $\mu \mathrm{m}$ (dominated by $\sim 100 \mu \mathrm{m}$ ). All the siltstone and sandstone rocks are fossil free and generally texturally immature. Framework-forming quartz and feldspar grains are mostly sub-angular to occasionally sub-rounded. Weathering-resistant accessory minerals, especially zircon, have a wider spectrum of roundness, from euhedral to well rounded. The sorting is generally moderate but is better in siltstone.

Mineralogically, quartz and muscovite are the dominant compounds in the sandstone and siltstone samples with $33-50 \%$ and $15-43 \%$, respectively. Among the heavy minerals, anatase and/or rutile were identified in all samples. The content of zircon is below sensitivity of the XRD analysis of ca. $0.5 \%$. Other components are variably represented (Table 1). The siltstone samples DR_DS, DR_HS1 and DR_HS2 from Drewer contain 2-5\% sodium plagioclase and $<1 \%$ potassium feldspar. Ankerite is the dominant carbonate. Pyrite is present in all studied samples but dominantly in the siltstone of the Drewer Sandstone. All the samples contain a small percentage of $1 \mathrm{M}$ illite of $1-6 \%$ but it is the 
Fig. 4 a Probability density plots of detrital zircon $\mathrm{U}-\mathrm{Pb}$ ages from four samples of top Famennian sandstone. b The youngest ages of the studied samples $<800 \mathrm{Ma}$. Neoprot. neoproterozoic, Mesoprot. mesoproterozoic, Paleoprot. paleoproterozoic
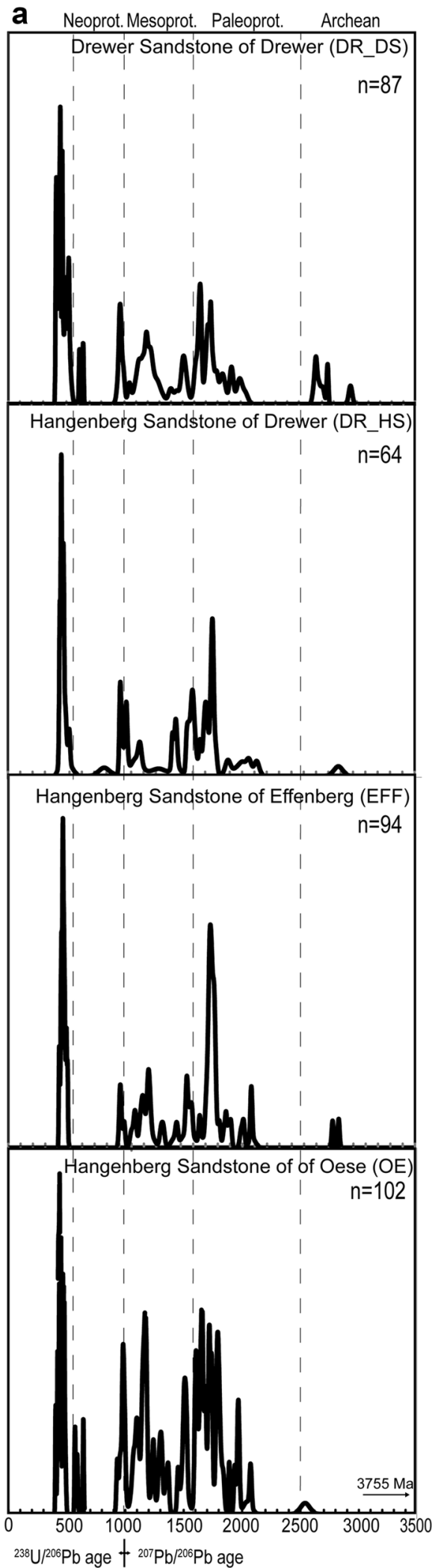

b

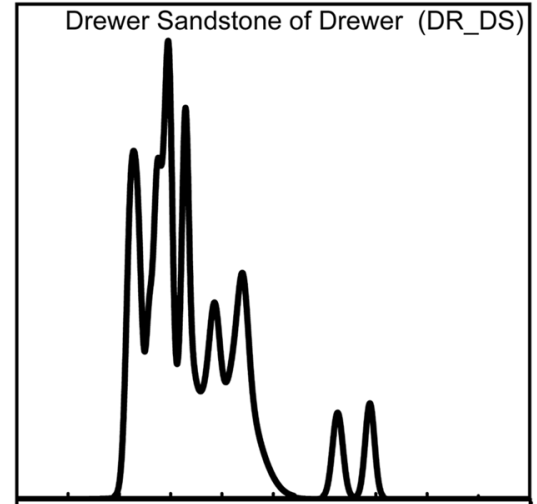

Hangenberg Sandstone of Drewer (DR_HS)

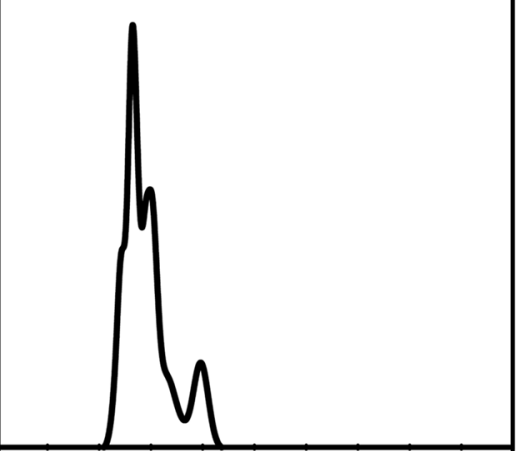

Hangenberg Sandstone of Effenberg (EFF)

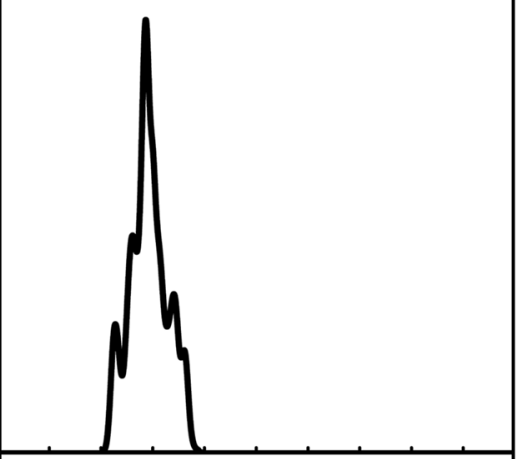

Hangenberg Sandstone of of Oese (OE)

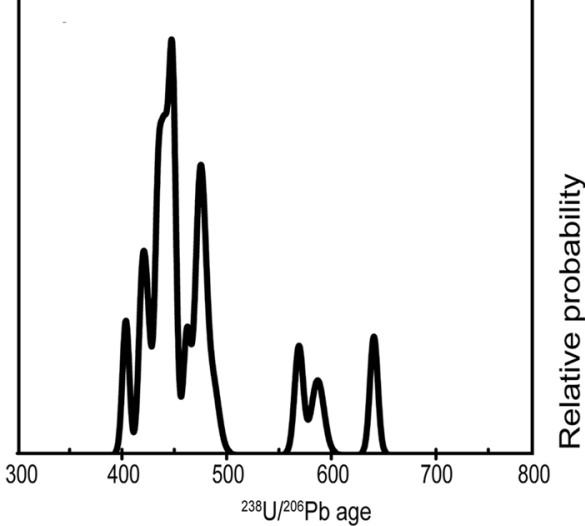

only clay mineral in DR_DS and DR_HS2. DR_HS 1 also contains $2 \%$ layered illite-smectite with an illitic component above ca. $80 \%$. The siltstone samples DR_DS and DR_HS from Drewer are quite inhomogenous, with laminae defined by the accumulation of muscovite flakes. The pyrite is present as framboidal crystals. 


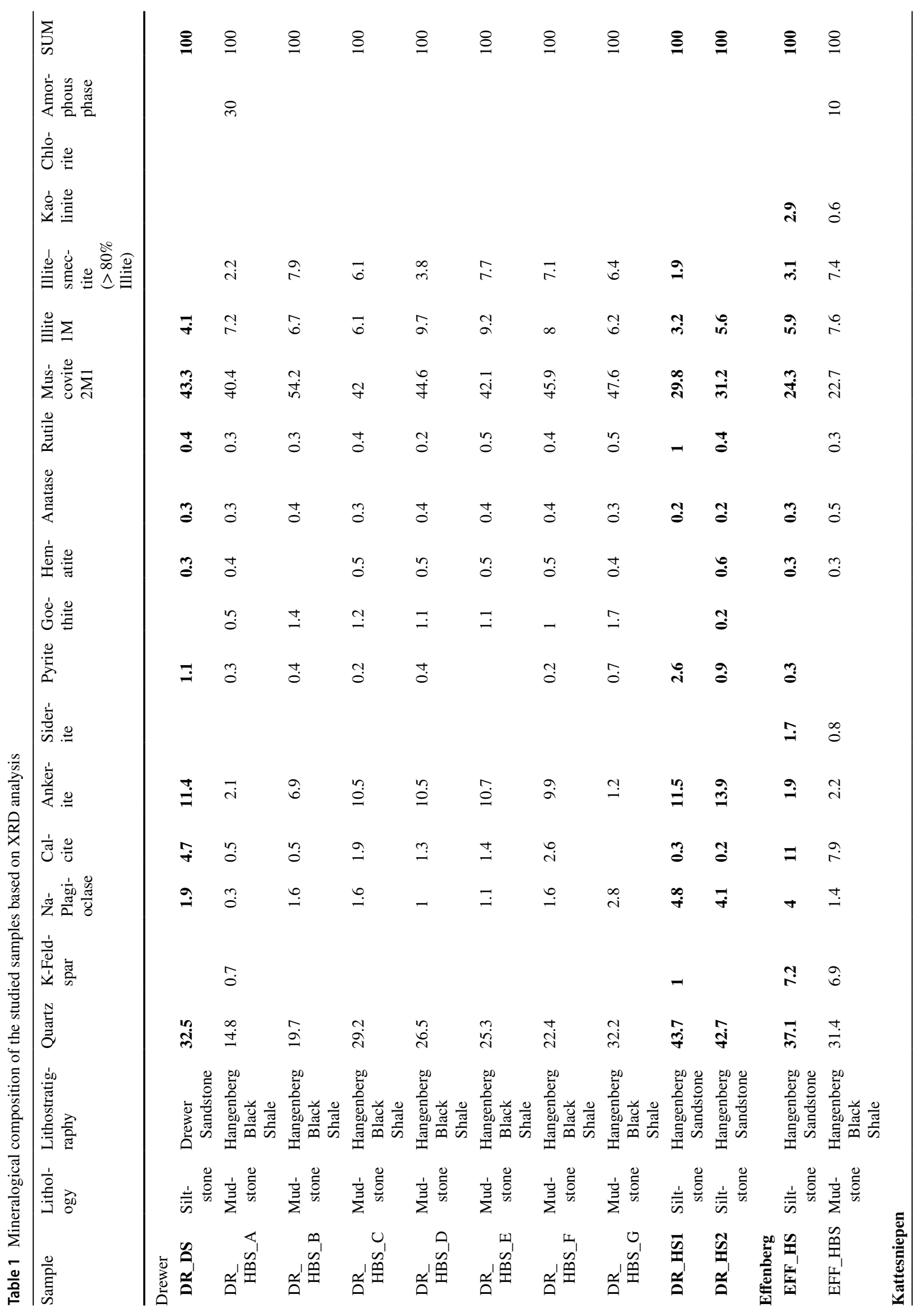




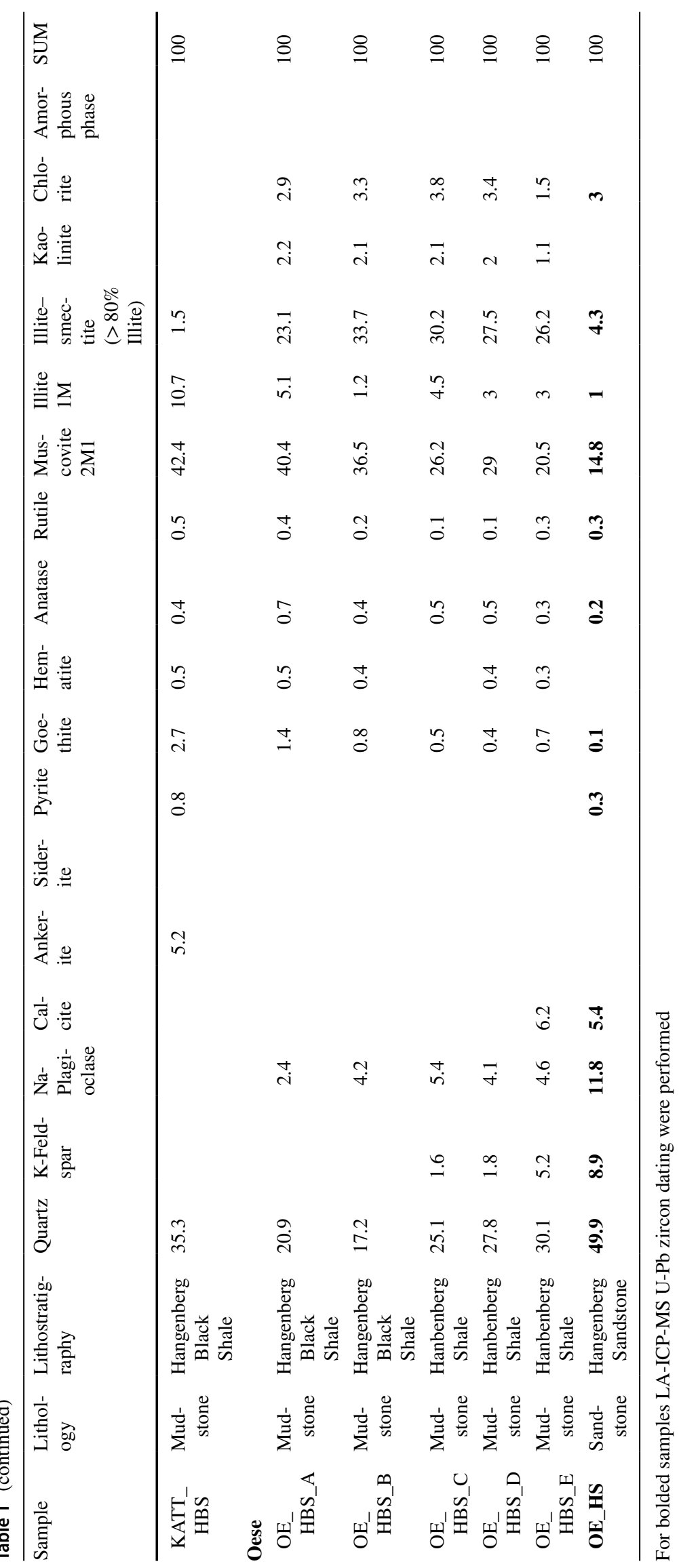


Differently to Drewer, the siltstone and sandstone from Effenberg (EFF_HS) and Oese (OE_HS) contain 7\% and 9\% potassium feldspar, respectively (Table 1 ). Calcite is the most common carbonate mineral with $11 \%$ and $5 \%$, respectively. The abundance of ankerite is less than $2 \%$. In addition to illite, the content of illite-smectite is 3 and $4 \%$, respectively. Furthermore, EFF_HS contains 3\% kaolinite and OF_HS 3\% chlorite. The Effenberg sample (EFF_HS) is heterolithic with an alternation of thicker siltstone and very thin mudstone layers. Chromian spinel was determined by SEM analysis of the heavy-mineral spectrum of the sandstone from the Hangenberg Sandstone (OE_HS; Fig. 5).

A similar general composition with dominance of quartz and muscovite and, locality-based modal composition for feldspar, carbonate and clay minerals is observed from the Hangenberg Black Shale and Hangenberg Shale from all studied localities (Table 1).

\section{Chemical composition}

The analyzed siltstone and sandstone display compositions with $\mathrm{SiO}_{2}$ contents of 56-75 wt.\% (average 62 $\pm 4 \%$ ), $\mathrm{Al}_{2} \mathrm{O}_{3}$ ranges from 10 to $13 \mathrm{wt}$. \% (average $12 \pm 1 \%$ ), $\mathrm{Na}_{2} \mathrm{O}$ from 0.4 to 1.6 wt. \% (average $0.7 \pm 0.3 \%$ ), $\mathrm{K}_{2} \mathrm{O}$ from 2.9 to $3.7 \mathrm{wt} . \%$ (average $3.2 \pm 0.2 \%$ ) and $\mathrm{Fe}_{2} \mathrm{O}_{3}$ contents of 2.4 to 5.5 wt. \% (average $4.2 \pm 0.8 \%$; Table 2; Fig. 6). Both the Hangenberg Black Shale and Hangenberg Shale are characterized by narrow compositional changes for $\mathrm{SiO}_{2}$, $\mathrm{Al}_{2} \mathrm{O}_{3}, \mathrm{~K}_{2} \mathrm{O}$ and $\mathrm{TiO}_{2}$, which have concentrations close to those of post-Archean Australian Shale (PAAS; Condie 1993; McLennan 2001; Table 2; Fig. 6). CaO shows highly variable concentrations ranging from 0.5 to 22.2 wt. \% (average $3.8 \pm 2.1 \%$ for the HBS and $7.6 \pm 5.2 \%$ for the Hangenberg Shale, respectively). $\mathrm{Fe}_{2} \mathrm{O}_{3}$ is also variable, with concentrations ranging from 2.4 to $7.2 \mathrm{wt} . \%$ (average $4.7 \pm 1.0 \%$ ). $\mathrm{Na}_{2} \mathrm{O}$ is strongly depleted relative to the upper continental crust (UCC; McLennan 2001) and PAAS (average $0.4 \pm 0.1 \%$; Fig. 6 ).

Most trace element concentrations $(\mathrm{Cr}, \mathrm{Ni}, \mathrm{Pb}, \mathrm{Sr}$, $\mathrm{Zn}, \mathrm{Zr}$ ) of the studied topmost Famennian rocks show higher values than those of the UCC and PAAS (Table 2; Fig. 6). Moreover, the Drewer samples are enriched in $\mathrm{Ba}$. The concentrations of $\mathrm{Ba}, \mathrm{Cr}, \mathrm{Cu}, \mathrm{Ni}, \mathrm{Pb}, \mathrm{V}$, and $\mathrm{Zn}$ show a poor correlation with $\mathrm{Al}(R \leq 0.5)$. In the studied samples, only $\mathrm{Ba}$ and $\mathrm{V}$ contents correlate with TOC (= total organic carbon) concentration $(R \geq 0.8)$, while $\mathrm{Cd}$ and $\mathrm{Zn}$ show positive correlation with $\mathrm{Fe}_{2} \mathrm{O}_{3}(R=0.7)$. A good correlation occurs between $\mathrm{Cr}$ and $\mathrm{TiO}_{2}, \mathrm{Cr}$ and $\mathrm{V}$ ( $R=0.6-0.7)$, while there is a poor correlation between $\mathrm{Cr}$ and $\mathrm{Al}(R=0.4)$. Rare earth element (REE) values and chondrite-normalized $\mathrm{La}_{\mathrm{N}} / \mathrm{Yb}_{\mathrm{N}}$ ratios, with average $8.3 \pm 0.6$ for the samples from the Drewer and Hangenberg Sandstones and 8.7 \pm 0.9 for mudstone of the HBS and Hangenberg Shale, are similar to those of the UCC and PAAS (Table 2; Fig. 6). The values of the Eu anomaly $\left(\mathrm{Eu} / \mathrm{Eu}^{*}\right)$ are $0.53-0.76$ (average $0.61 \pm 0.05$; Table 2 ) and they are slightly lower than the reference values of the UCC and PAAS. The provenance indicative element ratios $\mathrm{Th} / \mathrm{Sc}, \mathrm{Th} / \mathrm{Co}, \mathrm{La} / \mathrm{Sc}$ and $\mathrm{Cr} / \mathrm{Th}$ show higher values than those for the UCC for siltstone and sandstone, whereas mudstone ratios also lie above those for the PAAS (except $\mathrm{Th} / \mathrm{Sc}$; Table 2; Fig. 7). The values of $\mathrm{Zr} / \mathrm{Sc}$ and $\mathrm{Hf} / \mathrm{Sc}$ are 19-35 and 0.5-0.9, respectively, for sandstone and siltstone (Table 2), and 6-25 and 0.2-0.7, respectively, for mudstone.
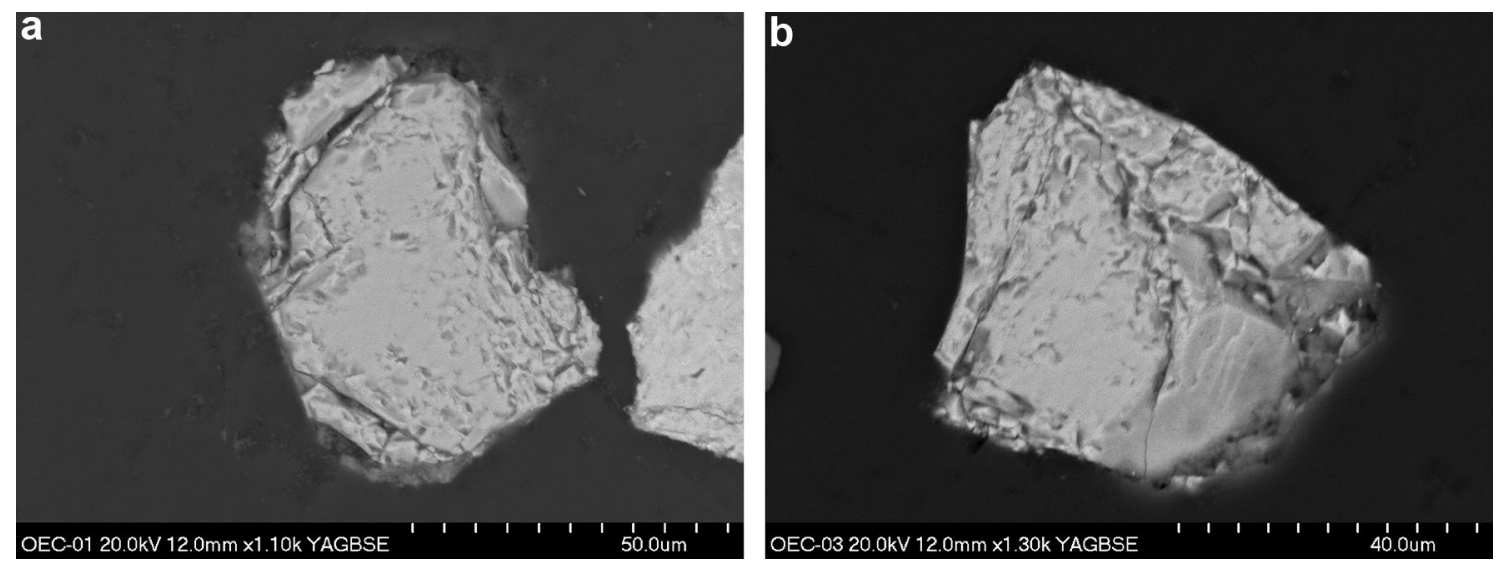

Fig. 5 Scanning electron microscope images showing morphology of chromian spinel from the sample OE_HS 


\begin{tabular}{|c|c|c|c|c|c|c|c|c|c|c|c|c|c|c|c|c|}
\hline 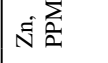 & in & g & $\Xi$ & $\Xi$ & $\stackrel{\infty}{=}$ & $\approx$ & $\stackrel{\varrho}{\Xi}$ & $\mathbb{N}$ & $\ddot{n}$ & $\nsubseteq$ & $\stackrel{\circ}{n}$ & 8 & $\simeq$ & $\stackrel{\infty}{\curvearrowright}$ & 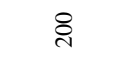 & $\stackrel{n}{r}$ \\
\hline$>>\frac{2}{2}$ & 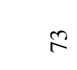 & $\stackrel{\circ}{\sim}$ & 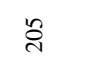 & $\vec{\square}$ & 导 & 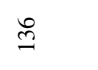 & $\tilde{n}$ & $\vec{n}$ & 5 & $\approx$ & సे & $\therefore$ & ปี & $\infty$ & $\stackrel{n}{n}$ & $\hat{m}$ \\
\hline$E \equiv$ & $=$ & $\simeq$ & $\underline{m}$ & 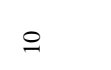 & 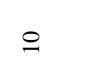 & 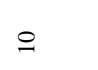 & $=$ & $\underline{m}$ & $=$ & $=$ & $\simeq$ & $=$ & \pm & $=$ & $\simeq$ & \pm \\
\hline 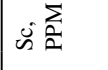 & $\simeq$ & $=$ & $\stackrel{\infty}{\sim}$ & \pm & $\cong$ & $\stackrel{\circ}{\circ}$ & 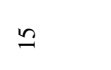 & 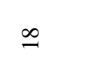 & 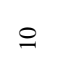 & $=$ & $=$ & $=$ & $\underline{m}$ & $a$ & $\stackrel{\circ}{\circ}$ & $=$ \\
\hline ف. & 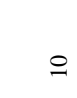 & in & F & $m$ & $m$ & $\approx$ & के & 亗 & $\stackrel{n}{\sim}$ & $=$ & in & $\stackrel{\infty}{\sim}$ & $\underline{m}$ & 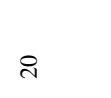 & $\ddot{3}$ & $\approx$ \\
\hline 语离 & 2 & \& & $\stackrel{\infty}{\gtrless}$ & $\mathscr{8}$ & 8 & $尺$ & $\approx$ & a & f & $\stackrel{\infty}{m}$ & $\exists$ & $\stackrel{\infty}{+}$ & 8 & $\stackrel{ \pm}{=}$ & $\vec{\infty}$ & $\stackrel{\circ}{\circ}$ \\
\hline 己ే & 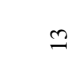 & r & $\vec{m}$ & $\tilde{\lambda}$ & $\stackrel{+}{\sim}$ & $\stackrel{+}{\sim}$ & \& & $\mathscr{F}$ & $=$ & $=$ & 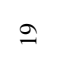 & $\stackrel{\infty}{\sim}$ & ส & $\stackrel{\infty}{+}$ & $m$ & $\stackrel{\infty}{\sim}$ \\
\hline 计离 & $\stackrel{m}{=}$ & 卞 & 导 & $n$ & $\stackrel{M}{\exists}$ & $\stackrel{M}{\exists}$ & 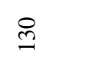 & ले & $\stackrel{t}{\subseteq}$ & $n$ & $\bar{\beth}$ & $\stackrel{9}{9}$ & $\stackrel{m}{=}$ & $n$ & 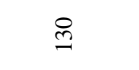 & $\stackrel{\infty}{\oplus}$ \\
\hline$\vec{u}$ & ชี & $\overrightarrow{0}$ & ? & $\stackrel{\circ}{\circ}$ & $n$ & $\stackrel{\circ}{\circ}$ & $\stackrel{\circ}{-}$ & $\overrightarrow{0}$ & $\stackrel{+}{0}$ & ฮี & ث̊. & 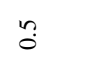 & $\overrightarrow{\hat{v}}$ & 3 & $\stackrel{m}{0}$ & 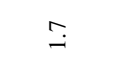 \\
\hline 赵 & প্ণ & $\stackrel{\text { 乌ิ }}{=}$ & $\stackrel{\tilde{z}}{\exists}$ & $\stackrel{\bar{g}}{\varrho}$ & ळू & $\stackrel{\partial}{\sigma}$ & $\widehat{\sigma}$ & 竎 & $\stackrel{\infty}{\infty}$ & ర్రి & $\stackrel{ \pm}{n}$ & $\stackrel{\infty}{\stackrel{\infty}{+}}$ & 年 & $\mathscr{J ్ ర}^{\infty}$ & ๙ & $\stackrel{\infty}{\underset{f}{f}}$ \\
\hline W & $\stackrel{8}{\circ}$ & 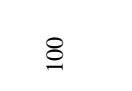 & $\stackrel{8}{\circ}$ & 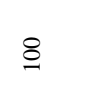 & 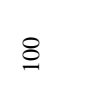 & $\stackrel{8}{\circ}$ & 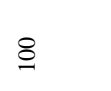 & $\stackrel{8}{\circ}$ & 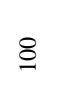 & 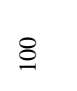 & 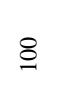 & 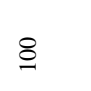 & 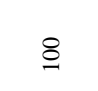 & 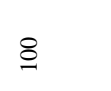 & 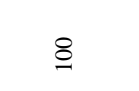 & 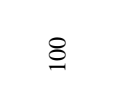 \\
\hline ô & $\overrightarrow{\mathrm{I}}$ & $\stackrel{2}{2}$ & $\begin{array}{l}\infty \\
\stackrel{\infty}{\omega}\end{array}$ & $\stackrel{\ddot{n}}{a}$ & $\begin{array}{l}n \\
n\end{array}$ & $\stackrel{\vec{n}}{ }$ & ñ & $\stackrel{\sigma}{\circ}$ & $\stackrel{m}{\varrho}$ & $\vec{\varrho}$ & $\stackrel{\infty}{\varrho}$ & $\stackrel{\infty}{a}$ & $\vec{r}$ & $\underset{\infty}{\infty}$ & $\stackrel{\circ}{=}$ & $\stackrel{g}{\dot{f}}$ \\
\hline $\begin{array}{l}0 \\
\Omega^{N}\end{array}$ & $\overrightarrow{0}$ & ชั & $\overrightarrow{0}$ & $\overrightarrow{0}$ & $\overrightarrow{0}$ & $\overrightarrow{0}$ & $\overrightarrow{0}$ & $\overline{0}$ & $\overline{0}$ & $\overrightarrow{0}$ & $\overline{0}$ & $\overrightarrow{0}$ & $\overrightarrow{0}$ & $\overrightarrow{0}$ & $\overrightarrow{0}$ & ฮั \\
\hline $\begin{array}{l}0 \\
\sum_{\Sigma}^{\Xi}\end{array}$ & $\stackrel{0}{0}$ & $\stackrel{8}{0}$ & $\stackrel{\infty}{\circ}$ & $\stackrel{\circ}{0}$ & $\stackrel{\infty}{0}$ & $\stackrel{\circ}{\circ}$ & $\stackrel{1}{0}$ & $\stackrel{n}{\circ}$ & $\stackrel{5}{0}$ & $\stackrel{\circ}{0}$ & $\stackrel{0}{0}$ & $\stackrel{5}{\circ}$ & 8 & $\stackrel{n}{\circ}$ & $\stackrel{8}{\circ}$ & $\stackrel{8}{\circ}$ \\
\hline$\overbrace{0}^{\circ}$ & $\stackrel{\infty}{\circ}$ & $\stackrel{\infty}{0}$ & $\stackrel{\infty}{0}$ & $\hat{o}$ & $\hat{o}$ & $\hat{o}$ & $\grave{o}$ & $\stackrel{\partial}{\circ}$ & $\hat{o}$ & $\hat{o}$ & $\hat{o}$ & $\hat{o}$ & $\stackrel{\infty}{\circ}$ & $\hat{o}$ & $\stackrel{\infty}{\circ}$ & $\stackrel{\circ}{-}$ \\
\hline 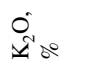 & $\hat{m}$ & $\dot{i}$ & $m_{n}^{m}$ & $\stackrel{?}{+}$ & $\stackrel{n}{+}$ & $\stackrel{f}{+}$ & $\stackrel{f}{+}$ & m & $\vec{m}$ & $\vec{m}$ & 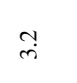 & $\ddot{\sim}$ & n & $\stackrel{m}{+}$ & $\stackrel{f}{f}$ & $n$ \\
\hline 䠌。 & t. & ฮุ & ? & $\stackrel{m}{0}$ & ? & $\stackrel{m}{0}$ & $\stackrel{3}{0}$ & ț & $\stackrel{\circ}{0}$ & $\stackrel{\circ}{0}$ & $n$ & $\stackrel{\circ}{0}$ & $\stackrel{n}{0}$ & $\because$ & $\overrightarrow{0}$ & 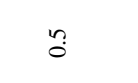 \\
\hline ๑ & $\stackrel{?}{r}$ & $m$ & $\stackrel{n}{+}$ & in & $\stackrel{+}{b}$ & in & $n$ & $n$ & $n$ & m & $\vec{b}$ & in & $\dot{m}$ & $\vec{b}$ & $\stackrel{\leftrightarrow}{i}$ & $\stackrel{\circ}{\circ}$ \\
\hline$\stackrel{\infty}{\infty}_{\Sigma}^{\infty}$ & $\hat{i}$ & ה̇ & $\stackrel{+}{i}$ & $\hat{i}$ & $\vec{m}$ & $\vec{i}$ & $n$ & $\cong$ & $\stackrel{\bullet}{i}$ & $\stackrel{n}{i}$ & $\stackrel{\infty}{i}$ & n & $\stackrel{\circ}{\circ}$ & $\stackrel{\Im}{=}$ & $\stackrel{\infty}{-}$ & $\ddot{i}$ \\
\hline $\begin{array}{l}w^{\prime} \\
w^{2}\end{array}$ & $\stackrel{\infty}{\oplus}$ & f & $\ddot{n}$ & $\underset{\dot{n}}{\stackrel{\infty}{i}}$ & $\begin{array}{l}\dot{0} \\
\dot{i n}\end{array}$ & in & $\stackrel{\circ}{i}$ & $n$ & $\stackrel{q}{q}$ & $\stackrel{n}{n}$ & $\stackrel{\mathcal{F}}{F}$ & $\stackrel{\circ}{\stackrel{f}{+}}$ & $\stackrel{+}{i}$ & $\stackrel{\circ}{\circ}$ & $\vec{i}$ & $\stackrel{ }{\sim}$ \\
\hline 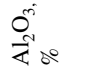 & $\stackrel{\mathrm{I}}{\mathrm{I}}$ & $\stackrel{\circ}{\stackrel{\infty}{\oplus}}$ & $\stackrel{\infty}{\stackrel{\infty}{\leftrightharpoons}}$ & $\stackrel{\circ}{\dot{ \pm}}$ & $\underline{m}$ & $\vec{\omega}$ & $\vec{\oplus}$ & $\overrightarrow{\underline{\infty}}$ & $\stackrel{\circ}{=}$ & $\stackrel{+}{=}$ & $\stackrel{\rho}{=}$ & $\stackrel{\circ}{=}$ & ตี & $\stackrel{\sim}{\subseteq}$ & $\stackrel{+}{\dot{\omega}}$ & $\stackrel{\text { กे }}{\mathrm{i}}$ \\
\hline$\overbrace{}^{\top}$ & $\vec{n}$ & $\stackrel{n}{7}$ & 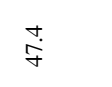 & ñ & $\underset{+\infty}{+\infty}$ & 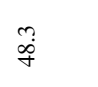 & $\begin{array}{c}m \\
\dot{\alpha} \\
\dot{\alpha}\end{array}$ & $\stackrel{m}{\infty}$ & $\tilde{\mathrm{B}}$ & 苞 & $\stackrel{n}{n}$ & $\vec{G}$ & $\tilde{B}$ & $\stackrel{\overbrace{}}{\vec{b}}$ & $\stackrel{m}{i n}$ & 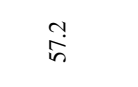 \\
\hline 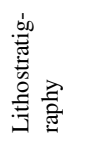 & & 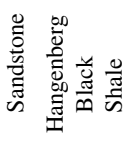 & 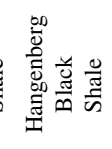 & 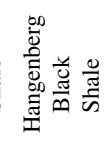 & 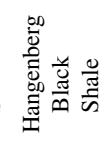 & 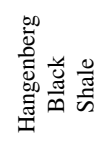 & 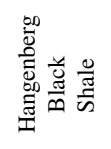 & 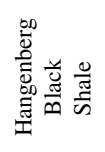 & 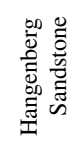 & 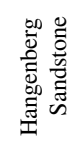 & 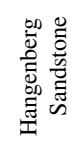 & 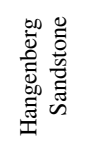 & 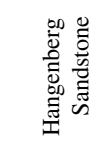 & 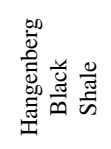 & 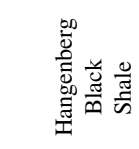 & 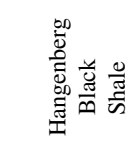 \\
\hline 立 & 站 & 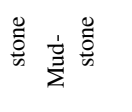 & $\sum_{\bar{\Sigma}}^{\dot{s}}$ & 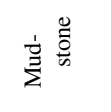 & 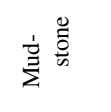 & $\sum_{\bar{\Sigma}}^{\dot{D}}$ & $\dot{\sum}^{\dot{s}}$ & $\sum_{\Sigma}^{\dot{\Xi}}$ & 咅 & 咅 & 站 & 站 & 咅 & 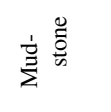 & $\dot{E}_{\Sigma}^{\stackrel{D}{E}}$ & $\sum_{\Sigma}^{\frac{1}{z}} \frac{0}{\frac{0}{n}}$ \\
\hline 20 & 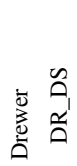 & 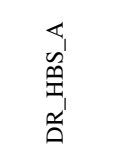 & 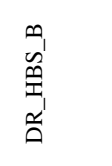 & 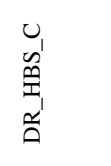 & 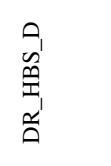 & 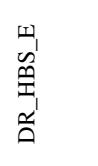 & 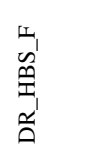 & $\begin{array}{l}0 \\
0 \\
0 \\
\tilde{\omega}_{1} \\
\stackrel{0}{0}\end{array}$ & $\begin{array}{l}\overrightarrow{\underline{n}} \\
\text { 足 } \\
\text { 定 }\end{array}$ & $\begin{array}{l}\tilde{\tilde{I}} \\
\underline{\Delta} \\
\tilde{\Delta}\end{array}$ & $\begin{array}{l}\tilde{\tilde{n}} \\
\hat{A}_{1} \\
\tilde{\Delta}\end{array}$ & $\begin{array}{l}n \\
0 \\
a \\
\tilde{a}\end{array}$ & 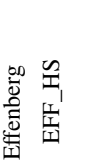 & 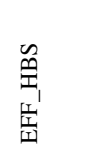 & 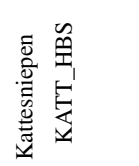 & 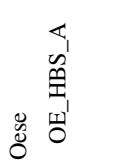 \\
\hline
\end{tabular}




\begin{tabular}{|c|c|c|c|c|c|c|c|c|c|c|c|c|c|c|c|c|c|c|}
\hline 촛 & $\tilde{b}$ & 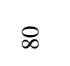 & తి & $\stackrel{\leftrightarrow}{\varrho}$ & $\stackrel{8}{8}$ & & 焦 & $\ddot{\infty}$ & లి & 8 & 8 & r & $\triangleright$ & $i n$ & in & $尺$ & $\tilde{v}$ & 6 \\
\hline & $\stackrel{ \pm}{I}$ & $\stackrel{\varrho}{\leftrightarrows}$ & $\hat{\sigma}$ & 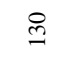 & ケ & & 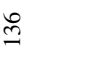 & 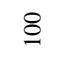 & $\stackrel{\infty}{\circ}$ & a & $\stackrel{\varrho}{\varrho}$ & $\stackrel{\Xi}{\Omega}$ & $\stackrel{\varrho}{\exists}$ & $\stackrel{\varrho}{9}$ & $R$ & 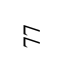 & $\alpha$ & $\stackrel{g}{g}$ \\
\hline$\dot{E} \bar{a}$ & 9 & 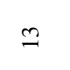 & 9 & $\simeq$ & $\infty$ & & \pm & 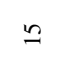 & 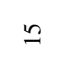 & \pm & $\Xi$ & 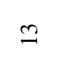 & $\Xi$ & $\stackrel{m}{M}$ & $a$ & $a$ & $\simeq$ & $=$ \\
\hline & $\stackrel{\bullet}{ }$ & $\stackrel{\circ}{\sim}$ & 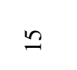 & $=$ & $r$ & & $=$ & 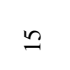 & $\underline{n}$ & $\stackrel{\bullet}{ }$ & $\cong$ & $=$ & $\stackrel{\infty}{\sim}$ & $\stackrel{\circ}{\sim}$ & $\simeq$ & $\simeq$ & $\underline{m}$ & $\stackrel{2}{ }$ \\
\hline 更 & f & $\stackrel{\infty}{m}$ & $\stackrel{\circ}{\circ}$ & 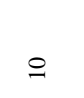 & $a$ & & $\infty$ & $\stackrel{\infty}{+}$ & i & $\stackrel{\infty}{i}$ & $\bar{\lambda}$ & $\grave{\lambda}$ & $\ddot{n}$ & $\stackrel{\infty}{\sim}$ & \pm & $\stackrel{m}{=}$ & $\stackrel{i}{i}$ & $=$ \\
\hline 证 & 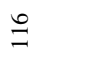 & $\approx$ & $\stackrel{t}{\stackrel{\Xi}{\sigma}}$ & q & $\stackrel{+}{d}$ & & 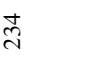 & $\stackrel{t}{ }$ & $\exists$ & t & 8 & in & 8 & 8 & से & $\stackrel{\infty}{\curvearrowright}$ & $\exists$ & $\exists$ \\
\hline 已ే & in & $\simeq$ & $\stackrel{\sim}{\sim}$ & in & $\simeq$ & & $\infty$ & $\tilde{m}$ & $\stackrel{\infty}{\sim}$ & $\stackrel{\infty}{\longrightarrow}$ & $\stackrel{\Delta}{\sim}$ & $\stackrel{i}{i}$ & $\stackrel{\infty}{\infty}$ & $\tilde{m}$ & $\stackrel{0}{-}$ & \pm & $\stackrel{\sim}{\sim}$ & $\stackrel{\infty}{\sim}$ \\
\hline & $\cong$ & $\Xi$ & $\stackrel{M}{=}$ & $\stackrel{\leftrightarrow}{=}$ & $\stackrel{\infty}{\curvearrowright}$ & & ఏ & $\stackrel{9}{\exists}$ & $\stackrel{\curvearrowleft}{\varrho}$ & 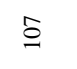 & $\Xi$ & $\vec{\sigma}$ & $\stackrel{ \pm}{\Xi}$ & $\stackrel{\infty}{=}$ & $\therefore$ & $F$ & $\infty$ & $\stackrel{\infty}{\beth}$ \\
\hline ઇే & $\stackrel{9}{9}$ & $\overrightarrow{\hat{v}}$ & 3 & $\overrightarrow{\hat{v}}$ & $\overrightarrow{\hat{v}}$ & & t. & 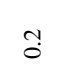 & $\overrightarrow{\hat{v}}$ & $\overline{0}$ & $\overrightarrow{\hat{v}}$ & ‡ & $\overrightarrow{\hat{v}}$ & $\stackrel{\leftrightarrow}{0}$ & ชั & $\overrightarrow{\hat{v}}$ & $\overrightarrow{\hat{v}}$ & $\overrightarrow{\hat{v}}$ \\
\hline פే & $\vec{\rho}$ & $\stackrel{\Im}{\exists}$ & $\stackrel{m}{m}$ & 诂 & $\stackrel{\infty}{\stackrel{\gamma}{\sigma}}$ & & f) & $\stackrel{\infty}{~}$ & $\stackrel{\infty}{m}$ & ફે & $\overrightarrow{\mathrm{D}}$ & : & ભે & ల్ల & 鿖 & $\tilde{\tilde{n}}$ & 导 & $\underset{q}{\sharp}$ \\
\hline W & 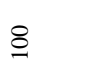 & 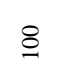 & 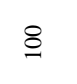 & $\stackrel{8}{-}$ & 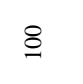 & & $\nsubseteq$ & 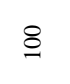 & 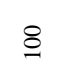 & 8 & 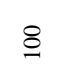 & 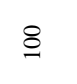 & 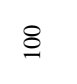 & 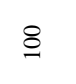 & $\stackrel{8}{\circ}$ & $\hat{a}$ & 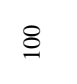 & 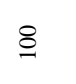 \\
\hline o & $\stackrel{\circ}{i}$ & $\ddot{q}$ & $\stackrel{?}{+}$ & $\stackrel{0}{0}$ & $\grave{n}$ & & $\overbrace{\infty}^{\infty}$ & $\stackrel{\bullet}{i}$ & $\stackrel{\bullet}{i}$ & $\stackrel{\infty}{\infty}$ & $\hat{\infty}_{\infty}$ & ڤุ & $a_{\infty}$ & $\stackrel{n}{\varrho}$ & $\overrightarrow{\dot{i}}$ & $\stackrel{\infty}{\varrho}$ & $\stackrel{?}{ \pm}$ & $\stackrel{n}{+}$ \\
\hline & ฮั & $\overline{0}$ & $\overrightarrow{0}$ & $\overrightarrow{0}$ & $\overrightarrow{0}$ & & ֻே & $\overrightarrow{0}$ & $\overline{0}$ & $\overrightarrow{0}$ & $\overrightarrow{0}$ & $\overrightarrow{0}$ & $\overline{0}$ & $\overrightarrow{0}$ & $\overrightarrow{0}$ & $\overrightarrow{0}$ & $\overrightarrow{0}$ & $\overline{0}$ \\
\hline & $\stackrel{\circ}{\circ}$ & $\stackrel{8}{\circ}$ & $\stackrel{t}{0}$ & $\stackrel{t}{0}$ & $\stackrel{\overbrace{}}{0}$ & & $\stackrel{9}{\circ}$ & $\stackrel{\leftrightarrow}{0}$ & $\stackrel{\circ}{\circ}$ & $\stackrel{d}{0}$ & $\stackrel{n}{0}$ & $\stackrel{n}{0}$ & ț & $\stackrel{t}{0}$ & $\stackrel{7}{0}$ & $\stackrel{\circ}{\circ}$ & $\stackrel{\circ}{0}$ & $\stackrel{n}{0}$ \\
\hline$\stackrel{\circ}{\because}$ & $\stackrel{8}{\circ}$ & $\stackrel{\circ}{-}$ & à & $\stackrel{\infty}{\circ}$ & $\stackrel{0}{0}$ & & ò & $\dot{\partial}$ & $\stackrel{\infty}{\circ}$ & $\stackrel{\infty}{\circ}$ & $\stackrel{\infty}{\circ}$ & $\stackrel{\infty}{\circ}$ & $\stackrel{\infty}{\circ}$ & $\stackrel{\infty}{\circ}$ & $n$ & $\because$ & $\hat{o}$ & ò \\
\hline & ir & $\vec{n}$ & $\stackrel{\curlyvee}{f}$ & † & $\vec{i}$ & & : & in & $\stackrel{q}{q}$ & 广 & $\stackrel{\circ}{+}$ & $\stackrel{\infty}{+}$ & in & $\stackrel{\infty}{+}$ & 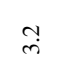 & $\stackrel{\circ}{\circ}$ & $\stackrel{+}{+}$ & ชુ \\
\hline & $n$ & $\hat{o}$ & $\hat{o}$ & $\stackrel{0}{\circ}$ & $\stackrel{\circ}{-}$ & & $\stackrel{+}{0}$ & $\hat{o}$ & $\stackrel{\circ}{\circ}$ & $?$ & $n$ & $\stackrel{t}{0}$ & ț & $\stackrel{3}{\circ}$ & ำ & ชุ & ?. & $\stackrel{m}{0}$ \\
\hline & $\stackrel{\infty}{\circ}$ & $n$ & $n$ & $\ddot{n}$ & $\stackrel{\infty}{i}$ & & $\stackrel{\infty}{i}$ & $\hat{i}$ & $\stackrel{0}{0}$ & $\stackrel{\infty}{0}$ & $\stackrel{m}{?}$ & $\stackrel{?}{?}$ & $\hat{b}$ & $\stackrel{a}{\infty}$ & $\overrightarrow{\mathrm{i}}$ & $\stackrel{\sim}{\sim}$ & $\stackrel{\circ}{\dot{I}}$ & $\dot{o}$ \\
\hline & ते & $\stackrel{\vec{i}}{\vec{i}}$ & $\vec{i}$ & $\stackrel{9}{9}$ & 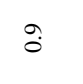 & & $\underset{\mathrm{i}}{\mathrm{i}}$ & $\Xi$ & 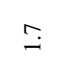 & $\stackrel{\infty}{-}$ & $\stackrel{\infty}{-}$ & 9 & $\stackrel{\leftrightarrow}{i}$ & $\stackrel{\infty}{-}$ & $\stackrel{m}{\rightarrow}$ & $\stackrel{n}{-}$ & $\stackrel{+}{\leftrightarrows}$ & $\stackrel{\infty}{-}$ \\
\hline 80 & $\stackrel{2}{2}$ & $\overrightarrow{i n}$ & $\stackrel{\infty}{i}$ & $\hat{n}$ & $\stackrel{\text { i }}{\mathrm{t}}$ & & i. & $\stackrel{\infty}{\Gamma}$ & $\stackrel{m}{m}$ & $\stackrel{\infty}{\sim}$ & $\vec{j}$ & $\stackrel{\text { I }}{\mathcal{T}}$ & $\stackrel{\circ}{+}$ & $\grave{j}$ & $\stackrel{\infty}{i}$ & $\stackrel{+}{i}$ & $\stackrel{\sim}{m}$ & $\stackrel{\circ}{+}$ \\
\hline . & ìं & $\bar{a}$ & $\stackrel{\sim}{\infty}$ & $\begin{array}{l}\stackrel{0}{\grave{g}}\end{array}$ & $\stackrel{\overbrace{}}{\varrho}$ & & ڤें & $\vec{\Xi}$ & 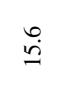 & $\stackrel{\overbrace{}}{\mathscr{\theta}}$ & in & 迥 & $\stackrel{+}{\stackrel{+}{\Xi}}$ & $\underset{ِ}{0}$ & $\exists$ & $\stackrel{\rho}{=}$ & $\stackrel{+}{ \pm}$ & $\stackrel{n}{2}$ \\
\hline & $n$ & $\frac{m}{6}$ & $\frac{9}{\dot{b}}$ & $\stackrel{m}{0}$ & $\stackrel{0}{\mathbb{I}}$ & & तี & $\stackrel{a}{\vec{b}}$ & $\stackrel{\circ}{\circ}$ & n. & $\begin{array}{l}0 \\
\dot{n}\end{array}$ & 官 & $\stackrel{0}{\dot{H}}$ & i̊ & $\underset{\infty}{\infty}$ & $\stackrel{\infty}{\infty}$ & $\hat{g}$ & $\vec{b}$ \\
\hline 咅 & 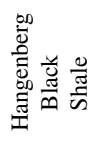 & 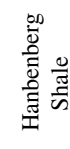 & 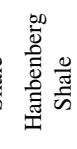 & 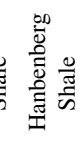 & 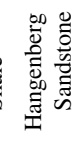 & . & 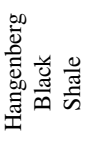 & 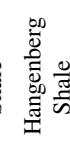 & 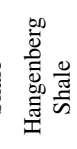 & 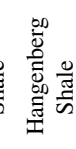 & 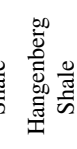 & 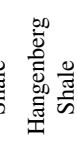 & 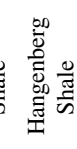 & 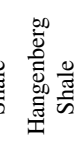 & 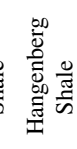 & 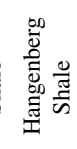 & 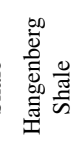 & 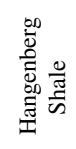 \\
\hline हुㅇํㅇ & 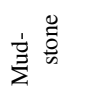 & $\sum_{\Sigma}^{\frac{1}{2}}$ & $\sum_{\bar{z}}^{\frac{1}{\bar{s}}}$ & $\sum_{2}^{\frac{1}{3}}$ & 宸 & 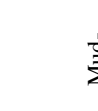 & 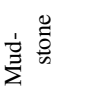 & 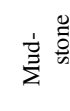 & $\frac{\dot{m}}{\sum^{2}}$ & $\frac{1}{2}$ & $\frac{0}{2}$ & $\frac{\partial}{2}$ & 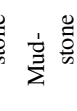 & $\frac{\partial}{\Sigma}$ & $\frac{0}{2}$ & $\frac{\dot{m}}{\sum_{2}}$ & 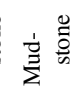 & $\dot{s}_{\dot{z}}$ \\
\hline 过 & 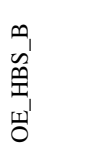 & 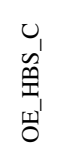 & 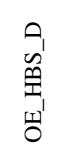 & 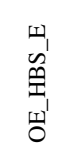 & $\begin{array}{l}\tilde{0}_{1} \\
\text { 빙 }\end{array}$ & 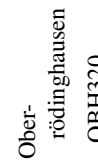 & 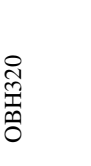 & 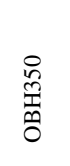 & 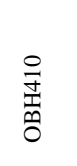 & 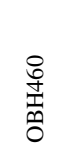 & $\begin{array}{l}8 \\
\text { : } \\
\text { 吾 } \\
0\end{array}$ & 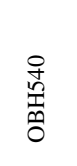 & 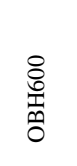 & 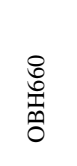 & 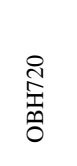 & 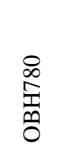 & 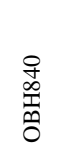 & 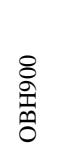 \\
\hline
\end{tabular}




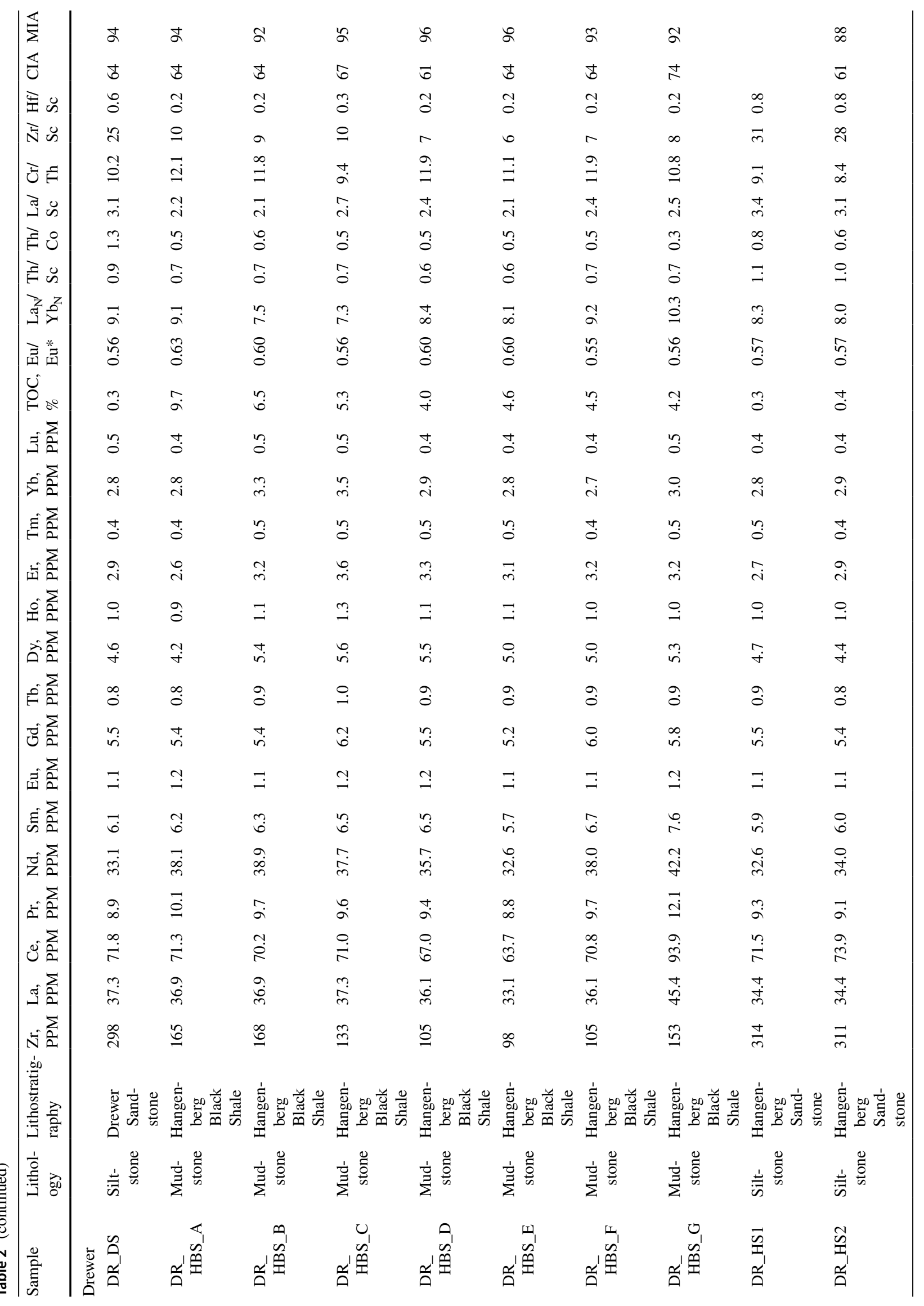




\begin{tabular}{|c|c|c|c|c|c|c|c|c|c|c|}
\hline$\S$ & $\bar{a}$ & & $\therefore$ & $\stackrel{2}{2}$ & 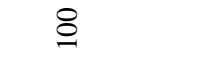 & \& & $\infty$ & $\stackrel{\infty}{\curvearrowright}$ & $\infty$ & $\cong$ \\
\hline च & $\vec{\sigma}$ & ช్ & $\vec{r}$ & 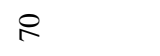 & $\vec{r}$ & $\mathbb{a}$ & $\mathbb{2}$ & $\mathbb{N}$ & $\mathbb{i}$ & i \\
\hline 再品 & $\tilde{o}$ & $\stackrel{\infty}{0}$ & $\tilde{0}$ & $\hat{o}$ & $\tilde{o}$ & $\stackrel{t}{0}$ & $\tilde{0}$ & $\stackrel{m}{0}$ & $\stackrel{t}{0}$ & $\because$ \\
\hline 今े & $\stackrel{\infty}{\sim}$ & নे & $\stackrel{2}{ }$ & $\ddot{n}$ & $a$ & $\cong$ & $a$ & 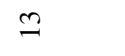 & $\cong$ & $\stackrel{\infty}{-}$ \\
\hline$\vec{U}$ & $\stackrel{\stackrel{\bullet}{0}}{\varrho}$ & $\stackrel{\infty}{=}$ & $\vec{\infty}$ & $\stackrel{\infty}{\infty}$ & $\hat{\varrho}$ & $\stackrel{\infty}{a}$ & $\stackrel{\circ}{\circ}$ & $\stackrel{\infty}{\infty}$ & $\stackrel{\infty}{\infty}$ & $\stackrel{\infty}{\infty}$ \\
\hline త్ & $\stackrel{\circ}{\dot{m}}$ & $\stackrel{m}{m}$ & $\underset{i}{0}$ & $\stackrel{\infty}{i}$ & $\stackrel{+}{i}$ & $\hat{\mathrm{i}}$ & $\tilde{\mathrm{H}}$ & $\tilde{n}$ & $\stackrel{\dot{m}}{\dot{m}}$ & $\stackrel{\bullet}{\dot{r}}$ \\
\hline झे & $\exists$ & $\dot{0}$ & ฮั & $\overrightarrow{0}$ & $\stackrel{t}{0}$ & $\stackrel{\Xi}{0}$ & กั & $\stackrel{\infty}{0}$ & $\stackrel{\infty}{0}$ & $\exists$ \\
\hline 引 & $=$ & $\stackrel{\circ}{-}$ & $\exists$ & $\stackrel{\text { Iִ }}{-}$ & $\stackrel{\infty}{0}$ & $\stackrel{\infty}{0}$ & $\stackrel{\infty}{0}$ & $\stackrel{\infty}{0}$ & $\stackrel{\infty}{0}$ & $\exists$ \\
\hline 产 & $\stackrel{\circ}{r}$ & $\stackrel{\infty}{r}$ & $\exists$ & $\stackrel{\circ}{\circ}$ & $\stackrel{\sim}{=}$ & $\begin{array}{l}\infty \\
\infty \\
\infty\end{array}$ & $\stackrel{\infty}{a}$ & $\stackrel{\infty}{r}$ & $\vec{\infty}$ & $\stackrel{\overbrace{}}{\varrho}$ \\
\hline 竎 & $\stackrel{n}{n}$ & $\tilde{n}$ & $\tilde{n}$ & $\stackrel{8}{\circ}$ & $\stackrel{n}{0}$ & $\stackrel{t}{0}$ & $\stackrel{0}{\stackrel{0}{0}}$ & $\stackrel{\circ}{\circ}$ & $\stackrel{\infty}{n}$ & $\stackrel{\infty}{\circ}$ \\
\hline$O_{6}$ & $\stackrel{t}{0}$ & $\tilde{3}$ & $\stackrel{t}{0}$ & $\stackrel{0}{0}$ & $\stackrel{\circ}{+}$ & $\tilde{3}$ & $n$ & $\tilde{3}$ & ֻั & $\tilde{3}$ \\
\hline$\exists=$ & $\stackrel{t}{0}$ & $n$ & 3 & 3 & $\stackrel{m}{0}$ & $?$ & $\stackrel{t}{0}$ & $n$ & $n$ & $\stackrel{t}{0}$ \\
\hline$\sum_{i} \sum_{\frac{1}{\alpha}}$ & $\hat{\mathrm{i}}$ & $\vec{m}$ & $\stackrel{\circ}{i}$ & $\stackrel{\infty}{-}$ & $\stackrel{m}{i}$ & $\stackrel{\infty}{\infty}$ & $\stackrel{\grave{i}}{\mathrm{i}}$ & $\stackrel{+}{m}$ & ले & $\stackrel{b}{i}$ \\
\hline 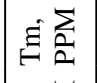 & t & $\because$ & 3 & $\stackrel{n}{0}$ & $\stackrel{+}{0}$ & $\stackrel{b}{0}$ & $\tilde{0}$ & $\tilde{0}$ & $\because$ & $\stackrel{t}{0}$ \\
\hline 武充 & $\hat{i}$ & $\stackrel{\sim}{n}$ & $\stackrel{\circ}{i}$ & $\stackrel{\infty}{-}$ & $\stackrel{m}{i}$ & $\stackrel{n}{n}$ & $\hat{i}$ & $\stackrel{\circ}{\dot{m}}$ & $\stackrel{\circ}{+}$ & $\vec{i}$ \\
\hline$\dot{\Phi}^{\circ}$ & $\ddot{\sigma}$ & $\rightrightarrows$ & $\stackrel{\circ}{0}$ & $\stackrel{0}{0}$ & $\hat{0}$ & $\stackrel{\Upsilon}{-}$ & $\stackrel{\circ}{-}$ & $\exists$ & $\stackrel{+}{-}$ & $\stackrel{\circ}{-}$ \\
\hline 交充 & $\stackrel{\circ}{i}$ & $\vec{n}$ & $\vec{i}$ & $\stackrel{\circ}{\dot{r}}$ & $\vec{n}$ & $\begin{array}{l}\dot{b} \\
\dot{n}\end{array}$ & $\stackrel{n}{\sim}$ & $\vec{n}$ & $\stackrel{\nabla}{b}$ & $\stackrel{\sim}{\sim}$ \\
\hline ही & $\stackrel{\infty}{\circ}$ & $\ddot{o}$ & $\ddot{0}$ & $\stackrel{0}{0}$ & $\stackrel{0}{\circ}$ & $\hat{o}$ & $\hat{o}$ & $\hat{o}$ & $\stackrel{m}{-}$ & $\stackrel{\infty}{0}$ \\
\hline లే & $\ddot{n}$ & $\stackrel{\infty}{\dot{n}}$ & $\stackrel{\vec{r}}{\dot{m}}$ & $\stackrel{\circ}{+}$ & $\stackrel{\bullet}{+}$ & $\ddot{i}$ & $\ddot{n}$ & $\ddot{n}$ & $\vec{\infty}$ & $\vec{n}$ \\
\hline 馬 & $\stackrel{\circ}{-}$ & $\stackrel{\sim}{-}$ & $\tilde{0}$ & $\stackrel{\infty}{0}$ & $a$ & $\stackrel{\Upsilon}{-}$ & $\stackrel{n}{-}$ & $\stackrel{m}{-}$ & 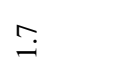 & $\stackrel{m}{-}$ \\
\hline 塱 & $\ddot{n}$ & రి & $\stackrel{\sim}{+}$ & $\stackrel{\bullet}{+}$ & ఫै. & $\stackrel{+}{b}$ & గี & $\hat{b}$ & $n$ & $n$ \\
\hline 宗充 & $\stackrel{\circ}{\dot{m}}$ & $\ddot{m}$ & $\dot{\vec{j}}$ & $\begin{array}{l}n \\
\infty \\
\infty\end{array}$ & $\hat{\dot{m}}$ & ڤे & ڤે & in & $\stackrel{\forall}{\dot{q}}$ & $\stackrel{\circ}{\dot{m}}$ \\
\hline 婄 & $\stackrel{\infty}{\infty}$ & ạ & $\stackrel{+}{\stackrel{十}{r}}$ & $\vec{r}$ & $\stackrel{\circ}{\varrho}$ & $\hat{\sigma}$ & 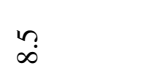 & ๙ุ & $\exists$ & $\hat{\infty}$ \\
\hline ن & $\stackrel{8}{\stackrel{2}{2}}$ & $\stackrel{\check{I}}{\stackrel{1}{*}}$ & $\vec{i}$ & $\hat{i}$ & $\tilde{\infty}$ & $\hat{i}$ & $\begin{array}{l}0 \\
\text { ¿̂. }\end{array}$ & $\overrightarrow{\dot{R}}$ & $\stackrel{+}{\dot{\infty}}$ & $\stackrel{\partial}{\hat{\sigma}}$ \\
\hline$\underset{\Xi}{\sum_{a}}$ & $\underset{\sim}{\stackrel{\sim}{i}}$ & 苍 & $\ddot{m}$ & $\vec{i}$ & $\begin{array}{l}m \\
\infty \\
\infty \\
\infty\end{array}$ & $\stackrel{\circ}{\dot{q}}$ & $\overrightarrow{\dot{m}}$ & $\stackrel{r}{\infty}$ & $\ddot{q}$ & $\overrightarrow{\dot{m}}$ \\
\hline 촐 & $\frac{m}{m}$ & 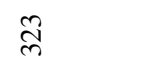 & 勇 & $\underset{\sim}{ }$ & I & $\stackrel{\mathscr{q}}{\mathrm{d}}$ & 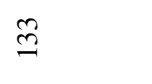 & ઠ્તે & $\widehat{ন}$ & હે \\
\hline 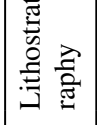 & 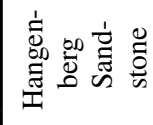 & 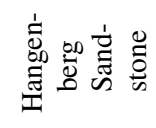 & 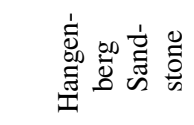 & 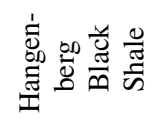 & 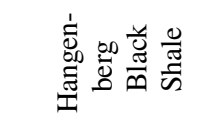 & 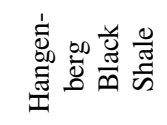 & 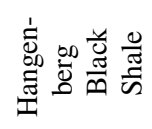 & 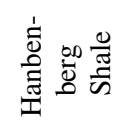 & 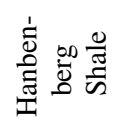 & 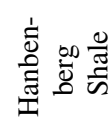 \\
\hline 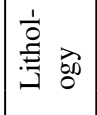 & 站 & 站 & 窝总 & 方总 & $\sum_{i}^{\frac{1}{2}}$ & 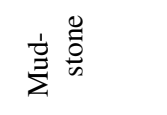 & 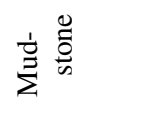 & $\sum_{\dot{z}}^{\dot{z}}$ & $\dot{\Xi}_{\Sigma}^{\dot{m}}$ & 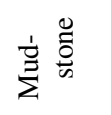 \\
\hline 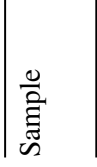 & $\begin{array}{l}\tilde{n} \\
\hat{\Omega}_{1} \\
\tilde{\theta}\end{array}$ & $\begin{array}{l}n \\
a_{1} \\
\tilde{a}\end{array}$ & 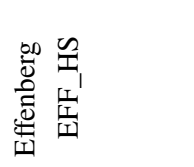 & 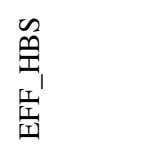 & 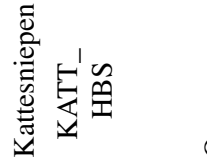 & 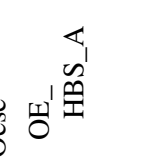 & $\begin{array}{c}\oplus^{\prime} \\
w^{\prime} \\
y^{\prime} \hat{l}^{0}\end{array}$ & 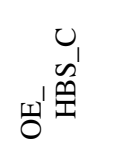 & 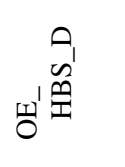 & 밍 \\
\hline
\end{tabular}




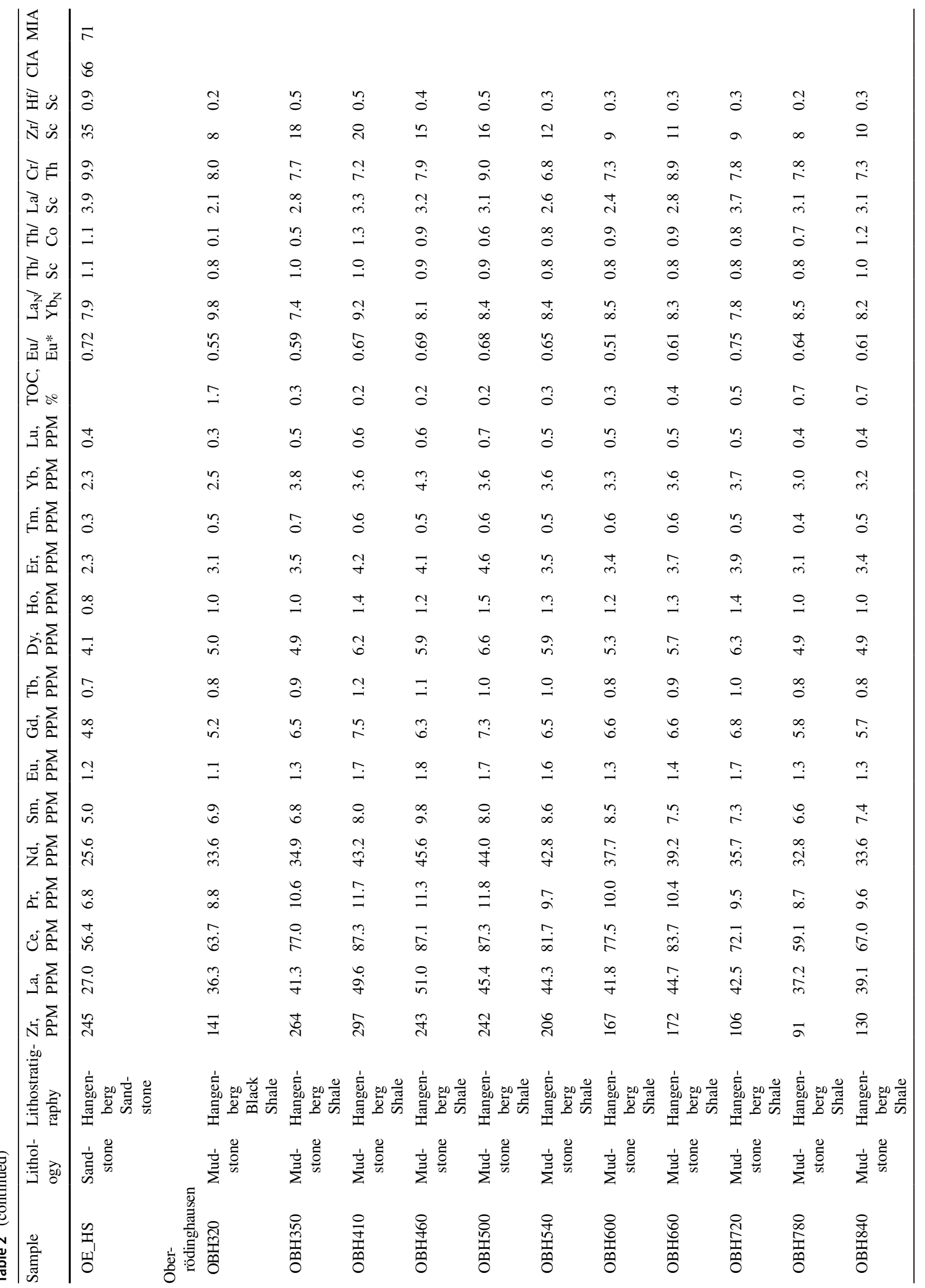




\section{Discussion}

\section{Paleo-weathering, sorting and recycling}

The comparison of the mineralogical data from the Hangenberg Sandstone samples with data from Lower and Upper Devonian sediments from the RWTH-1 drill hole (northern Eifel region near Aachen, northwestern Rhenish Massif; Sindern et al. 2008) shows higher abundances of ankerite, illite and detrital muscovite and less plagioclase, $\mathrm{K}$-feldspar and chlorite in the uppermost Famennian samples from the northern Rhenish Massif. The sandstone and siltstone of the Hangenberg Sandstone have a more similar mineralogical composition to the Upper Carboniferous siliciclastic rocks of the northwestern Rhenish Massif than to Lower and Upper Devonian sedimentary rocks (Table 1 in Sindern et al. 2008). The $\mathrm{SiO}_{2}, \mathrm{Al}_{2} \mathrm{O}_{3}$, $\mathrm{K}_{2} \mathrm{O}$ and $\mathrm{Fe}_{2} \mathrm{O}_{3}$ contents are similar to values of Upper Devonian siliciclastic rocks in the northwestern Rhenish Massif (Sindern et al. 2008) but different from the major element composition of the Middle-Upper Devonian Giessen greywacke (Floyd et al. 1991) of the southeastern Rhenish Massif.

The topmost Famennian sedimentary rocks of the northern Rhenish Massif have been affected by a moderate degree of chemical alteration (a proxy of the intensity of weathering), as indicated by the chemical index of alteration values of 61-71 (sandstone and siltstone) and 61-74 (mudstone), respectively (Fig. 8; Nesbitt and Young 1982). A higher degree of alteration is given by the mineralogical index of alteration with MIA $_{\text {sandstone and siltstone }}=71-94$ and MIA $_{\text {mudstone }}=75-96$. This is mainly due to very low feldspar contents (Fig. 8; except sample OE_HS). The CIA and MIA values in studied Drewer and Hangenberg Sandstones, Hangenberg Black Shale and Hangenberg Shale samples are different from the values of older Famennian siltstone and sandstone (considered as an equivalent of the Condroz Sandstone; Fig. 3) and the upper Famennian Shale of the northwestern Rhenish Massif close to Aachen (CIA $=43-74$, average 62; Sindern et al. 2008). The CIA and MIA values of the studied sedimentary rocks suggest intense weathering in the source area under steady-state conditions during which the material removal rate matches the production of mineralogically uniform weathering products (Nesbitt et al. 1997). Higher plagioclase and K-feldspar contents (Plag + K-fsp) of the sandstone and siltstone of the Hangenberg Sandstone in the Oese (20.7\%) and Effenberg (11.2\%) sections indicate the delivery of less weathered detritus in comparison to the highly matured mineralogical composition of the siltstone of the Drewer and Hangenberg Sandstones at Drewer (1.9 and $5.0 \%$, respectively; Table 1). Generally, the topmost 
Fig. 6 Major, trace and REE element compositions of $\mathbf{a}$ sandstone and siltstone of the Drewer and Hangenberg Sandstones and $\mathbf{b}$ mudstone of the Hangenberg Black Shale and Hangenberg Shale normalized to average upper continental crust (UCC; McLennan 2001)

The post-Archean Australian Shales (PAAS; Condie 1993; McLennan 2001) composition is shown for comparison

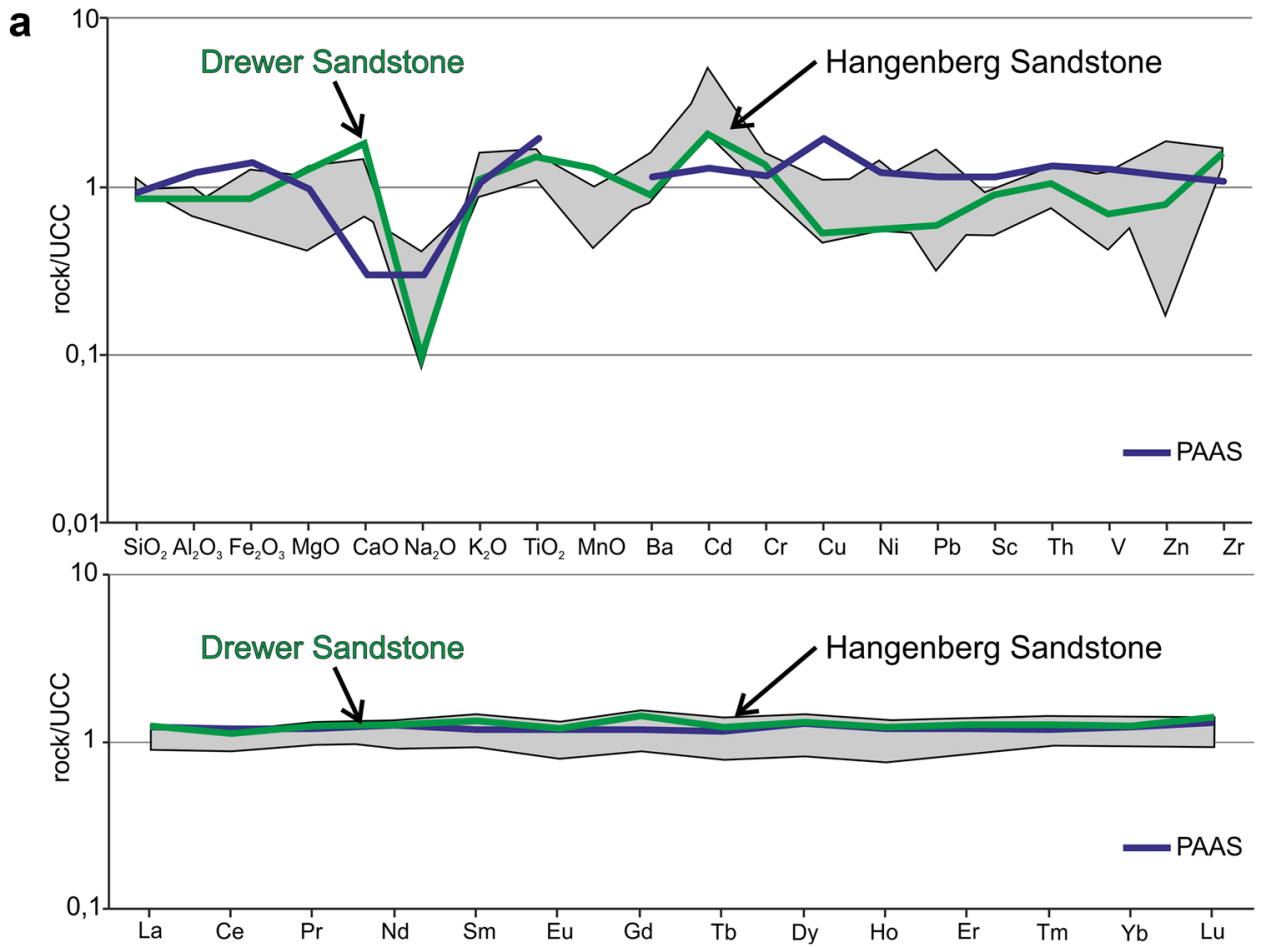

b
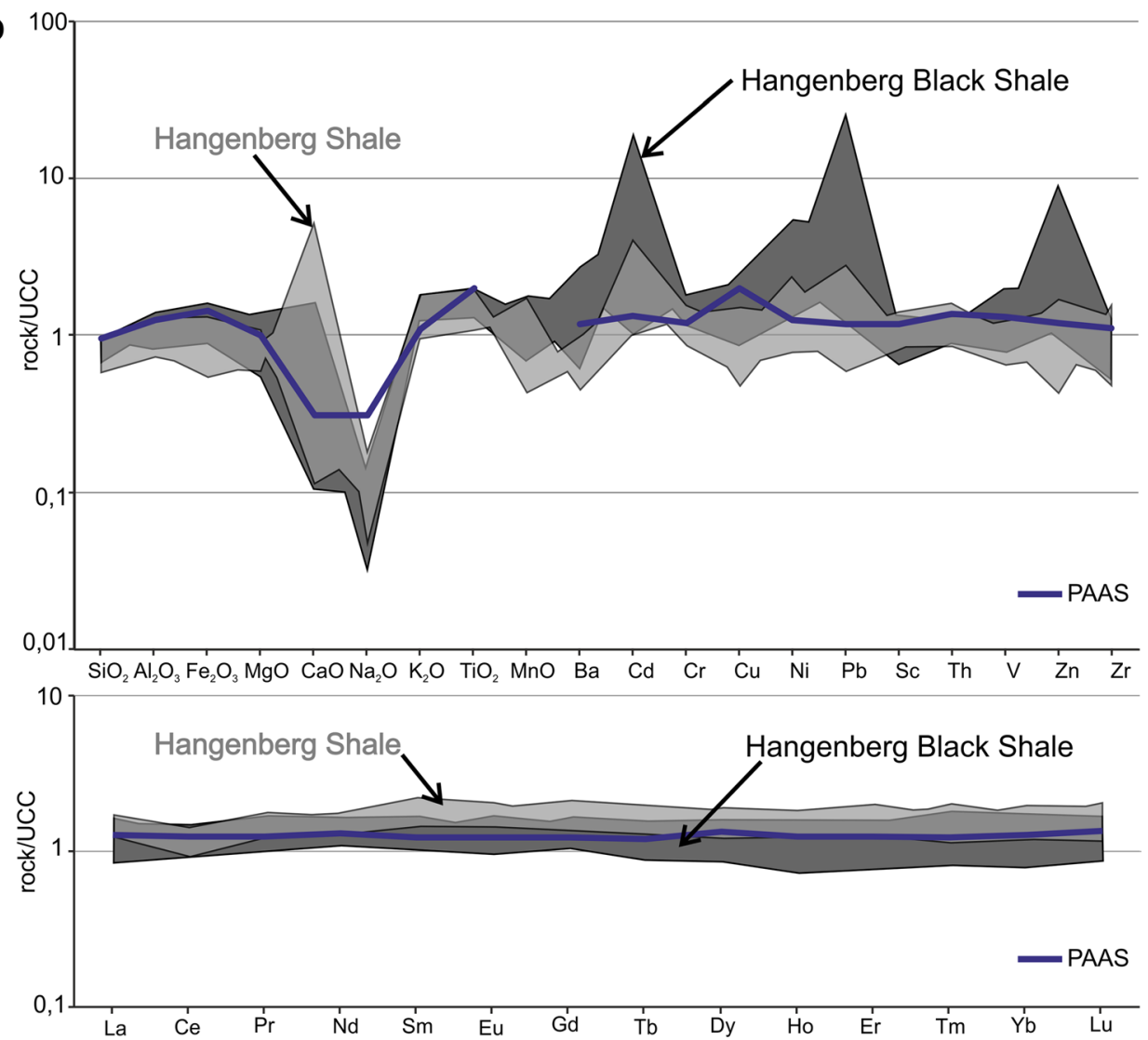

Famennian sedimentary rocks of the northern Rhenish Massif show a higher degree of chemical and mineralogical alterations than the older Famennian terrigenous sedimentary rocks of the northwestern Rhenohercynian Basin (Sindern et al. 2008). 


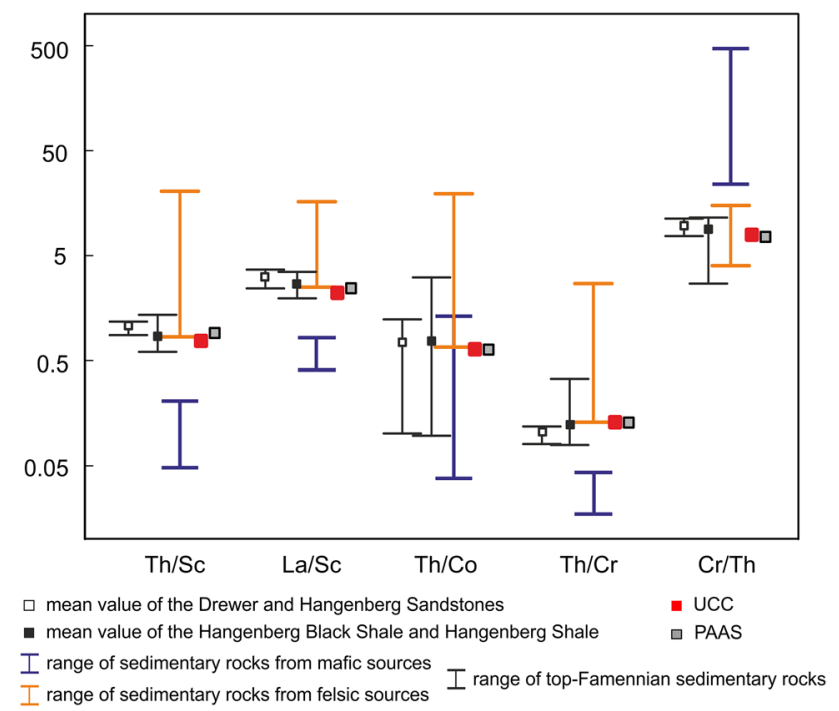

Fig. 7 Variations of $\mathrm{Th} / \mathrm{Sc}, \mathrm{La} / \mathrm{Sc}, \mathrm{Th} / \mathrm{Co}, \mathrm{Th} / \mathrm{Cr}$ and $\mathrm{Cr} / \mathrm{Th}$ ratios in the siliciclastic sedimentary rocks of the Drewer and Hangenberg Sandstones, Hangenberg Black Shale and Hangenberg Shale of the northern Rhenish Massif. The upper continental crust (UCC; McLennan 2001) and the post-Archean Australian Shales (PAAS; Condie 1993; McLennan 2001) composition is shown for comparison

Similar $\mathrm{Cr}, \mathrm{Cu}$, and $\mathrm{Zr}$ concentrations of the studied sedimentary rocks were reported from Upper Devonian siltstone, sandstone and shale of the Rhenohercynian Basin (Schulz-Dobrick and Wedepohl 1983; Sindern et al. 2008). The Giessen greywacke shows similar Ba, Hf, Ni, Zn and $\mathrm{Zr}$ concentrations (Floyd et al. 1991) as the Hangenberg Sandstone. The contents of $\mathrm{Cd}$ and $\mathrm{Ba}$ in some Famennian siltstone and mudstone from the Rhenish Massif are the highest among Upper Devonian siliciclastic rocks from the Rhenohercynian Basin (Schulz-Dobrick and Wedepohl 1983; Floyd et al. 1991).

$\mathrm{Ba}, \mathrm{Cr}, \mathrm{Cu}, \mathrm{Ni}, \mathrm{Pb}, \mathrm{V}$ and $\mathrm{Zn}$ are essential micro-nutrients for phytoplankton and thus intimately related to the growth of oceanic phytoplankton. Organic matter-rich mudstone, such as the Hangenberg Black Shale, deposited in anoxic and sulfidic conditions is typically enriched in trace elements associated with organic carbon burial fluxes (e.g., $\mathrm{Cd}, \mathrm{Cu}$ ) and/or with sulphides (e.g., Ni, Zn; Tribovillard et al. 2006). Studies of concentrations of $\mathrm{Zn}$ in iron formations (Robbins et al. 2013) revealed that elevated $Z n$ values may reflect a local $\mathrm{Zn}$ enrichment in near-vent hydrothermal exhalative sediments. Similarly, high $\mathrm{Cu}, \mathrm{Fe}$ and Ba concentrations indicate a contribution from hydrothermal sources (e.g., Robbins et al. 2013; von Raumer et al. 2017). Therefore, although the enrichment of $\mathrm{Ba}, \mathrm{Zn}, \mathrm{Cu}, \mathrm{Ni}, \mathrm{Cd}$, and TOC reflects the primary productivity in the Hangenberg Black Shale (Kaiser et al. 2015), it cannot be excluded that elevated concentrations were induced by hydrothermal processes connected with terminal Devonian synsedimentary volcanism (e.g., Iberian Pyrite Belt; Oliveira et al. 2005) or later, during postdepositional Variscan deformation, as in other parts of the northwestern Rhenohercynian Basin (Sindern et al. 2008).

The relatively high contents of $\mathrm{Cr}$ and $\mathrm{Ni}$ are typical for Lower and Middle Devonian sedimentary rocks of the Rhenish Massif that are indicative of basic and ultrabasic rocks and ophiolite in the source regions of these sedimentary rocks (Schulz-Dobrick and Wedepohl 1983; Press 1986). The elevated $\mathrm{Cr}$ concentrations of up to $130 \mathrm{ppm}$ for the siltstone and $147 \mathrm{ppm}$ for the mudstone are in accordance with the Hangenberg siliciclastic rocks containing detrital chromian spinel, as observed is OE_HS,

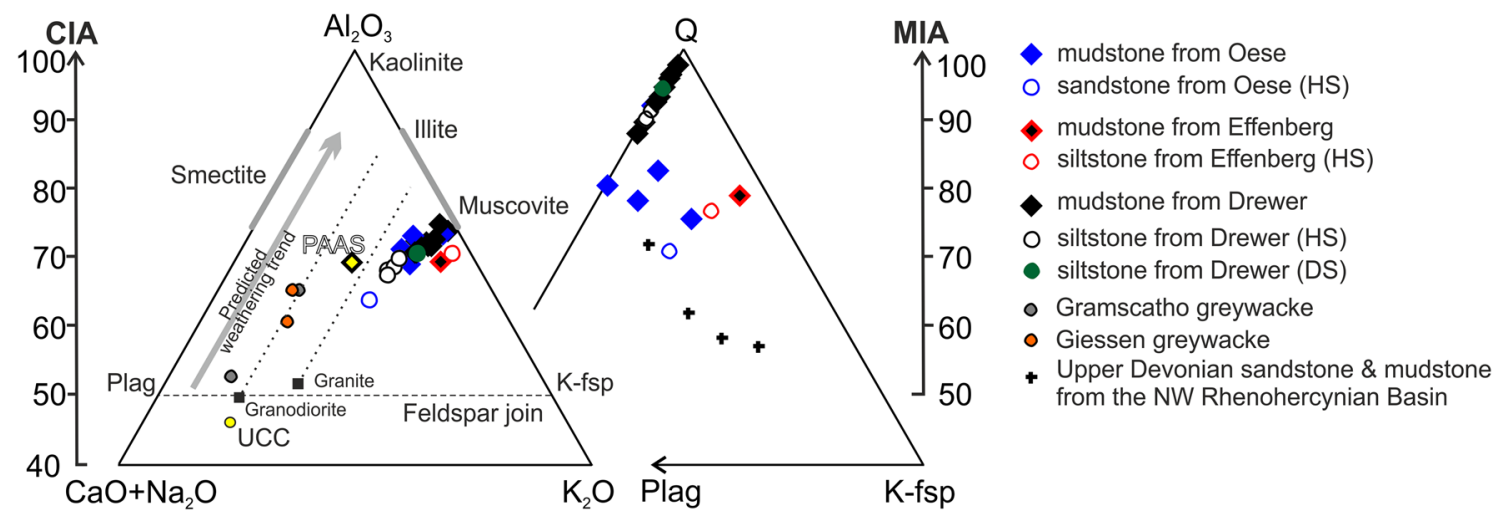

Fig. 8 Mineralogical and chemical composition of samples of the Drewer and Hangenberg Sandstones, Hangenberg Black Shale and Hangenberg Shale plotted in quartz (Q), plagioclase (Plag), potassium feldspar (K-fsp) diagram and in $\mathrm{Al}_{2} \mathrm{O}_{3}-\mathrm{CaO}^{*}+\mathrm{Na}_{2} \mathrm{O}-\mathrm{K}_{2} \mathrm{O}$ compositional space (Nesbitt and Young 1984). Note that the lower part of the diagrams with $\mathrm{Al}_{2} \mathrm{O}_{3}$ and $\mathrm{Q}<40$ is not shown. MIA is mineralogical index of alteration $(\mathrm{MIA}=$ [quartz/(quartz $+\mathrm{K}$-feld- spar + plagioclase $) \times 100$ ) and CIA (Nesbitt and Young 1982; Nesbitt et al. 1997) is chemical index of alteration (CIA $=\left[\mathrm{Al}_{2} \mathrm{O}_{3}\right.$ / $\left.\left.\left(\mathrm{Al}_{2} \mathrm{O}_{3}+\mathrm{CaO}^{*}+\mathrm{Na}_{2} \mathrm{O}+\mathrm{K}_{2} \mathrm{O}\right)\right] \times 100\right)$. Middle to Upper Devonian Gramscatho and Giessen Greywacke data after Floyd et al. (1991); Famennian sandstone and mudstone data from the RWTH-1 well (northwestern Rhenohercynian close to Aachen; Sindern et al. 2008) are shown for comparison 
Fig. 9 La-Th-Sc plot (Bhatia and Crook 1986) for Famennian silt-, sand- and mudstone from the northern Rhenish Massif. The areas of typical rock types after Perri et al. (2012). The Gramscatho and Giessen Greywacke data are after Floyd et al. (1991). The upper continental crust after McLennan (2001), the post-Archean Australian Shales after Condie (1993) and McLennan (2001)

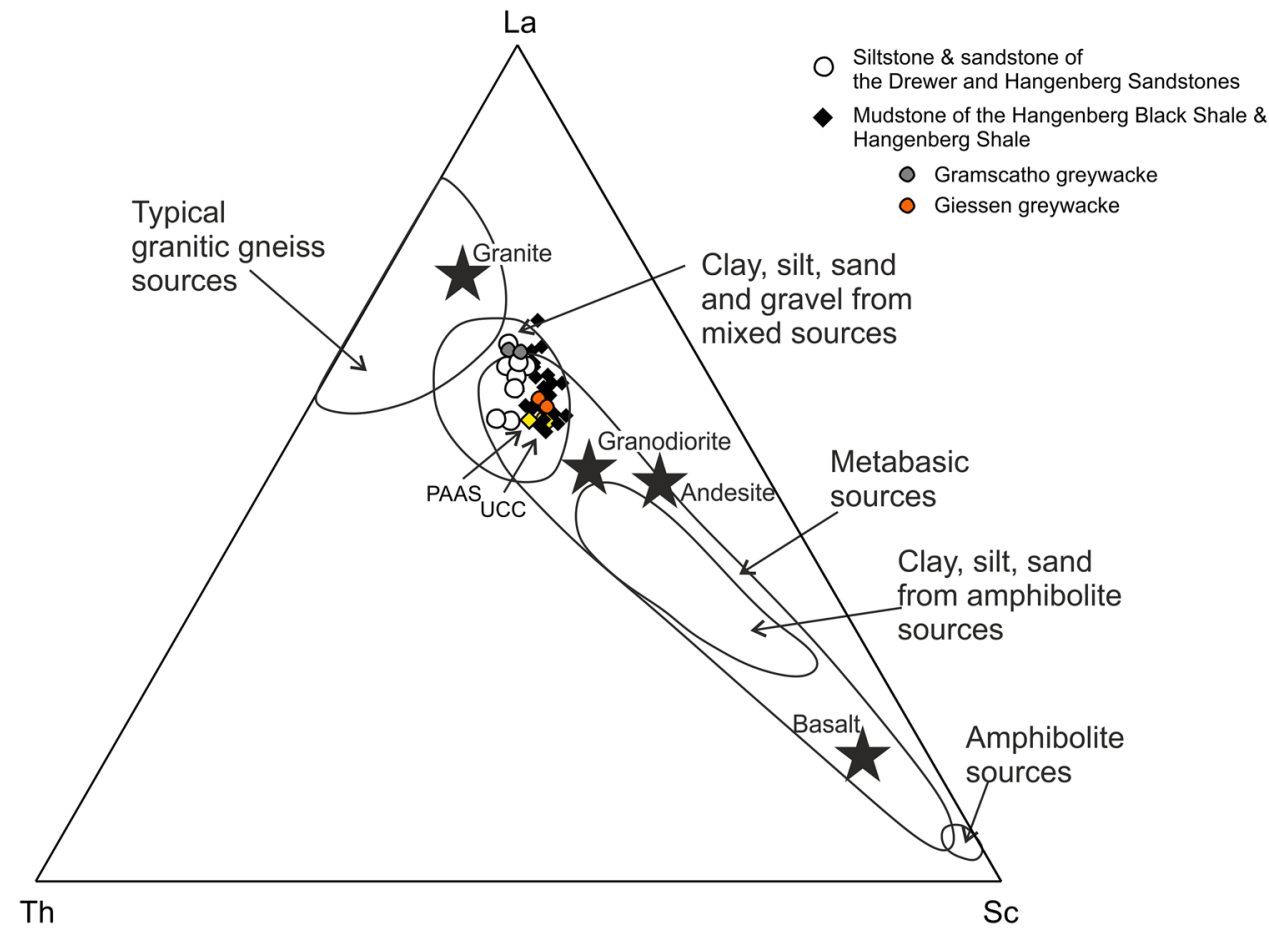

as well as in Lower Devonian sedimentary rocks of the northern Eifel Mountains (Haverkamp 1991; Fig. 1b) and Middle Devonian siltstone and sandstone of the Rhenish Massif (Press 1986). Furthermore, Middle Devonian sandstone of the Rügen Depression in northeastern Germany has elevated Cr values (McCann 1999), likely genetically linked with chromian spinel grains. A probable primary crystalline source of the detrital chromian spinel is preGivetian Alpine-type ophiolite (Press 1986; Sindern et al. 2008) of the Middle-Upper Ordovician Rügen greywacke (Giese et al. 1994). Hence, Cr spinel originally most probably derived from uplifted Caledonian orogen parts and from the exposed parts of Baltica in the north and northeast from where it was transported into Rhenohercynian Basin during the Lower-Middle Devonian (Schulz-Dobrick and Wedepohl 1983; Press 1986). The source terrain could be located in the Arkona High (Rügen area; Northeastern Germany) inside the Caledonide deformation zone, where Mesozoic strata directly cover Lower Paleozoic rocks of the Caledonian structural stage (without Upper Paleozoic sedimentary units; Katzung et al. 1993). The presence of $\mathrm{Cr}$ spinel in the Hangenberg Sandstone suggests that sediment of the northern part of the Rhenohercynian Basin still derived from a northern source area during the terminal Devonian and they could have been recycled from Ordovician Rügen greywacke. Recycling from the greywacke is supported by the rounded zircon grains in the studied samples that indicate a long-range detritus transport paths or/ and input of detritus from recycled sources. This is further supported by the high $\mathrm{Zr} / \mathrm{Sc}$ and $\mathrm{Hf} / \mathrm{Sc}$ values.

\section{Detrital zircon provenance}

The oscillatory zoning and high $\mathrm{Th} / \mathrm{U}$ in the detrital zircon indicate that originally most of them were sourced from magmatic rocks. The uniform $\mathrm{La}_{\mathrm{N}} / \mathrm{Yb}_{\mathrm{N}}$ ratios suggest that the sediment had a common source or several ones with mixing being efficient during transport and deposition. The provenance proxies suggest that the detritus originated mainly from continental, felsic material (Fig. 7; compare Augustsson and Bahlburg 2008). More specifically, the geochemical characteristics point towards sources with a granitic to granodioritic composition (Fig. 9). On the other hand, the presence of chromian spinel indicates also mafic to ultramafic rocks in the primary (first order) crystalline source.

In accordance with the geochemical composition, the uniform zircon age distributions of the studied rocks also indicate a similar felsic source for all samples. The small differences in zircon age distributions may be attributed to grain size sorting and break-up of zircon during sediment erosion, transport and re-sedimentation (Sláma and Kosler 2012). Our new zircon ages indicate that the top-Famennian detrital material was probably not derived from southern sources, as proposed in the past (Paproth 1986). There are several lines of evidence indicating that the transport of detritus to the Rhenish Massif was rather from the northern sources. First, the dominance of Mesoproterozoic and Paleoproterozoic zircon is typical for Baltica-derived grains. The range of ages corresponds to those known from magmatic rocks of Sveconorwegian and Svecofennian ancestry 
and of the Transscandinavian Igneous Belt, occupying large extents of Norway, Sweden, Poland, Denmark, and northern Germany (e.g., Söderlund et al. 1999; Andersen et al. 2002; Bingen et al. 2003).

Secondly, the other prominent detrital zircon group of Cambrian-Silurian age (500-400 Ma) matches transport from the Caledonian orogen of Scandinavia (e.g., Gee and Sturt 1985; Rey et al. 1997). The geographically closer German-Polish (Rügen-Pomeranian) Caledonides are considered as minor source area only due to limited availability. Devonian siliciclastic rocks from Pomerania in northwestern Poland contain detrital zircon with Caledonian crystallization ages and detrital muscovite with mostly Caledonian cooling ages (Paszkowski, unpublished data). This indicates that the German-Polish Caledonides were not fully submerged during the Middle Devonian as postulated e.g., by Franke (1989), but that small shoals/highs composed of Ordovician sequences and Caledonian magmatic rocks were subjected to erosion. Such structural highs have been confirmed by geophysical studies in the Rügen area with the spinel-rich greywacke (e.g., Piske et al. 1994; Hoffmann et al. 1998). This is in accordance with data from the Devonian of Pomerania (northwestern Poland), which is known to have been sourced from German-Polish Caledonides and where Caledonian zircon grains represent a smaller proportion of the detrital zircon population than in our samples (Paszkowski, unpublished data). Additionally, Caledonian ages are also known from the Silurian magmatic arc in the MidGerman Crystalline High and the Northern Phyllite Zone in the south (Fig. 1a; Franke 2000; Zeh and Will 2010; Franke and Dulce 2017). However, the dominance in our samples of Mesoproterozoic and Paleoproterozoic zircon ages that is typical for Baltica indicates that also the Caledonian zircons were most probably derived from northern sources. Besides, the Mid-German Crystalline High and the Northern Phyllite Zone contain a restricted population of Caledonian ages not older than $\sim 430 \mathrm{Ma}$, at odds with the wide range of Caledonian ages of ca 400-500 Ma found in the studied sedimentary rocks. Derivation from southern sources also can be ruled out due to the lack of Neoproterozoic and Cambrian (700-500 Ma) zircon. The 700-500 Ma component is characteristic of Devonian and Lower Carboniferous sedimentary rocks from the allochthonous units in the southern Rhenish Massif that were fed from the northern margin of the Saxothuringian Zone bearing Gondwana zircon signatures (Eckelmann et al. 2014). Potential other southern clastic sources are a system of shoals that developed during regressions in the central to northern Rhenish Massif area (e.g., cores of the future Ebbe, Remscheid-Altena and Siegen anticlines; Paproth 1986), from which recycled detrital material with Baltica signatures could have supplied Famennian siliciclastic rocks. However, the scarcity in the studied sedimentary rocks of ca. $500 \mathrm{Ma}$ ages typical for the Lower
Devonian from the Ebbe Anticline (Wagener 1989) provides further evidence against a southern transport. Also, zircon of Lower/Middle Devonian (Emsian and Eifelian) age, which is present in the Siegen and Ebbe anticlines and related to the wide-spread keratophyre volcanism (Loske and Miller 1987; Winter 2006), is missing in the studied samples. Besides, the narrow, synsedimentary active Rhenohercynian Basin formed during the Upper Devonian a trap for detritus from southern areas.

For the studied siliciclastic rocks, a derivation of detritus from the Avalonian basement of the Rhenish Massif can also be ruled out. This is supported by the lack of Neoproterozoic age, which are typical for Avalonian sources (Linnemann et al. 2012) in the Drewer and Hangenberg Sandstones. Small Middle/Upper Devonian exposures of Caledonian basement occurred also in the present-day subsurface northwestern Rhenish Massif, from where detritus including phyllite and quartzite was shed into Givetian neritic to reefal and Frasnian/lower Famennian post-reefal successions (e.g., Ribbert 1982; Neumann-Mahlkau and Ribbert 1998; Ahrendt et al. 2001). It is currently unknown, whether these very local deposits share the Avalonian signatures.

Our interpretation is in line with that of Zeh and Gerdes (2010), who concluded a northern Baltica source of detritus for the Rhenohercynian Basin, from the upper Silurian to the Upper Devonian/Lower Carboniferous. Detrital zircon populations with Baltican affinities are known from Lower Devonian to Upper Carboniferous sandstone in other parts of the Rhenish Massif (Haverkamp et al. 1992; Huckriede et al. 2004; Eckelmann et al. 2014). Such similarities in detrital zircon age distribution suggest that the transport of detritus from the north was not a local event but rather a regional phenomenon that lasted through the Devonian/ Carboniferous.

\section{Conclusions}

Hydrothermal processes connected with terminal Devonian synsedimentary volcanism or post-depositional Variscan deformation are the likely reason for $\mathrm{Ba}, \mathrm{Cu}, \mathrm{Pb}$, and $\mathrm{Zn}$ enrichments in topmost Devonian sedimentary rocks of the northwestern Rhenish Massif as well as in some other sedimentary sequences in the Rhenish Massif.

The chemical and mineralogical composition of the studied siltstone and sandstone indicate an advanced stage of sediment maturity, with detritus derived predominantly from felsic sources. The detrital chromian spinel in the Hangenberg Sandstone indicates mafic to ultramafic rocks in the source area or, more probably, the recycling of uplifted Caledonian orogen parts, and/or were derived from the exposed parts of Baltica in the north and northeast. 
Based on detrital zircon spectra, the studied top-Famennian siliciclastic sedimentary rocks from the northern Rhenish Massif have Baltic provenance signature with a majority of Paleo- and Mesoproterozoic ( 2000-1000 Ma) and lower Paleozoic ages ( 500-400 Ma) of Scandinavian Caledonides affinity, and the Rügen-Pomeranian Caledonides (Arkona High) as a minor contributor to the detritus budget. In conclusion, our provenance study enabled to settle previous speculations regarding the source areas for Upper Devonian rock of the northern Rhenish Massif.

Acknowledgements We thank M. Rakociński, D. Korn and M. Banaś for help during fieldwork, Z. Ciesielska and T. Siwecki for the preparation of samples for mineralogical and petrographic analyses, and $\mathrm{M}$. Plech and A. Łaptaś for assistance in the preparation of several drawings. We gratefully acknowledge the helpful and constructive reviews by Carita Augustsson (Stavanger) and by an anonymous reviewer. This study was supported by the National Science Centre in Poland research Grant No. 2011/03/B/ST10/04602. Some work was supported by the National Science Centre in Poland Grants Nos. 2011/01/B/ST10/01106 and 2013/08/A/ST10/00717.

Open Access This article is distributed under the terms of the Creative Commons Attribution 4.0 International License (http://creativeco mmons.org/licenses/by/4.0/), which permits unrestricted use, distribution, and reproduction in any medium, provided you give appropriate credit to the original author(s) and the source, provide a link to the Creative Commons license, and indicate if changes were made.

\section{References}

Ahrendt H, Ribbert KH, Vanguestaine M, Wemmer K (2001) K-Ar and acritarch dating of phyllite clasts from a re-sedimented Middle Devonian conglomerate in the northwestern part of the Rhenish Slate Mountains. Z Dtsch Geol Ges 152:365-377

Andersen T, Andresen A, Sylvester AG (2002) Timing of lateto postorogenic Sveconorwegian granitic magmatism in the Telemark and Rogaland-Vest Agder sectors, S. Norway. Norges Geol Unders Bull 440:5-18

Augustsson C, Bahlburg H (2008) Provenance of late Palaeozoic metasediments of the Patagonian proto-Pacific margin (southernmost Chile and Argentina). Int J Earth Sci 97:71-88

Becker RT (2008) Devonian neritic-pelagic correlations-methods, case studies and problems. In: Königshof P, Linnemann U (eds) 20th International Senckenberg Conference and 2nd Geinitz-Conference: "From Gondwana and Laurussia to Pangaea: Dynamics of Oceans and Supercontinents", Frankfurt am Main, September 30-October 3, 2008, Abstracts and Programme. (Frankfurt a M, Dresden), 25-28

Becker RT, Korn D, Paproth E, Streel M (1993) Beds near the Devonian-Carboniferous boundary in the Rhenish Massif, Germany. IUGS Subcommission on Carboniferous Stratigraphy (SCCS), Guidebook. Services assodociés de paléontologique de 1`ULg, Liége, pp 1-85

Becker RT, Kaiser SI, Aretz M (2016a) Review of chrono-, litho- and biostratigraphy across the global Hangenberg Crisis and Devonian-Carboniferous Boundary. In: Becker RT, Königshof P, Brett CE (eds) Devonian Climate, Sea Level and Evolutionary Events. Geological Society, London, Special Publications, vol 423, pp. 355-386
Becker RT, Aboussalam ZS, Stichling S, May A, Eichholt S (2016b) The Givetian-Frasnian Hönne valley reef complex (northern Sauerland) — an outline of stratigraphy and facies development. Münstersche Forsch Geol Paläontologie 108:126-140

Becker RT, Hartenfels S, Weyer D, Kumpan T (2016c) The Famennian to Lower Visean at Drewer (northern Rhenish Massif). Münstersche Forsch Geol Paläontologie 108:158-178

Bełka Z, Devleeschouwer X, Narkiewicz M, Piecha M, Reijers TJA, Ribbert KH, Smith NJP (2010) Devonian. In: Doornenbal JC, Stevenson AG (eds) Petroleum Geological Atlas of the Southern Permian Basin Area. EAGE Publications b.v. (Houten), pp 71-79

Bhatia MR, Crook KAW (1986) Trace element characteristics of greywackes and tectonic setting discrimination of sedimentary basins. Contrib Miner Petrol 92:181-193

Bingen B, Nordgulen $\varnothing$, Sigmond EMO, Tucker R, Mansfeld J, Hogdahl K, Norway S (2003) Relations between 1.19-1.13 Ga continental magmatism, sedimentation and metamorphism, Sveconorwegian province. Precambr Res 124:215-241

Bless M, Becker RT, Higgs K, Paproth E, Streel M (1993) Eustatic cycles around the Devonian-Carboniferous boundary and the sedimentary and fossil record in Sauerland (Federal Republic of Germany). Ann Soc Géol Belg 115:689-702 (imprint 1992)

Brinkmann R (1948) Die Mitteldeutsche Schwelle. Geol Rundsch $36: 56-66$

Condie KC (1993) Chemical composition and evolution of the upper continental crust: Contrasting results from surface samples and shales. Chem Geol 104:1-37

Corfu F, Hanchar JM, Hoskin POW, Kinny P (2003) Atlas of zircon textures. In: Hanchar JM, Hoskin, P.W.O. [eds], Zircon. Reviews in Mineralogy and Geochemistry 53: 469-500

Crônier C (2007) Larval morphology and ontogeny of an Upper Devonian phacopid: Nephranops from Thuringia, Germany. J Paleontol 81:684-700

Dörr W, Zulauf G, Gerdes A, Loeckle F (2017) Provenance of Upper Devonian clastic (meta)sediments of the Böllstein Odenwald (Mid-German-Crystalline-Zone, Variscides). Int J Earth Sci 106:2927-2943

Eckelmann K, Nesbor HD, Königshof P, Linnemann U, Hofmann M, Lange JM, Sagawe A (2014) Plate interactions of Laurussia and Gondwana during the formation of Pangaea-Constraints from U-Pb LA-SF-ICP-MS detrital zircon ages of Devonian and Early Carboniferous siliciclastics of the Rhenohercynian zone, Central European Variscides. Gondwana Res 25:1484-1500

Floyd PA, Shail R, Leveridge BE, Franke W (1991) Geochemistry and provenance of Rhenohercynian synorogenic sandstones: implications for tectonic environment discrimination. Geol Soc Lond Spec Publ 57:173-188

Franke W (1989) Tectonostratigraphic units in the Variscan belt of Central Europe. Special Papers. Geol Soc Am 230:67-90

Franke W (1995) Rhenohercynian foldbelt: autochthon and nonmetamorphic nappe units-stratigraphy. In: Dallmeyer D, Franke W, Weber K (eds) Pre-Permian Geology of Central and Eastern Europe. Springer, Berlin, pp 33-39

Franke W (2000) The mid-European segment of the Variscides: tectonostratigraphic units, terrane boundaries and plate tectonic evolution. Geol Soc Lond Spec Publ 179:35-61

Franke W (2014) Topography of the Variscan orogen in Europe: failednot collapsed. Int J Earth Sci 103:1471-1499

Franke W, Dulce JC (2017) Back to sender: tectonic accretion and recycling of Baltica-derived Devonian clastic sediments in the Rheno-Hercynian Variscides. Int J Earth Sci 106:377-386

Franke W, Engel W (1986) Synorogenic sedimentation in the Variscan belt of Europe. Bulletin de la Société Géologique de France 2(1):25-33 
Franke W, Oncken O (1990) Geodynamic evolution of the northcentral Variscides-a comic strip. In: Freeman R, Giese P, Mueller S (eds) The European Geotraverse. European Science Foundation, pp 187-194

Franke W, Oncken O (1995) Zur prädevonischen Geschichte des Rhenohercynischen Beckens. Nova Acta Leopoldina Neue Folge 71:53-72

Franke W, Cocks LRM, Torsvik TH (2017) The Palaeozoic Variscan oceans revisited. Gondwana Res 48:257-284

Gee DG, Sturt BA (1985) The Caledonide orogeny-Scandinavia and related areas. Wiley, Chichester, pp 1-1266

Geisler T, Vinx R, Martin-Gombojav N, Pidgeon RT (2005) Ion microprobe (SHRIMP) dating of detrital zircon grains from quartzites of the Eckergneiss Complex, Harz Mountains (Germany): implications for the provenance and the geological history. Int J Earth Sci 94:369-384

Giese U, Katzung G, Walter R (1994) Detrital composition of Ordovician sandstones from the Rügen boreholes: implications for the evolution of the Tornquist Ocean. Geol Rundsch 83:293-308

Grad M, Guterch A, Mazur S (2002) Seismic refraction evidence for crustal structure in the central part of the Trans-European Suture Zone in Poland. Geol Soc Lond Spec Publ 201:295-309

Hahn HD (1990) Fazies grobklastischer Gesteine des Unterdevons (Graue Phyllite bis Taunusquarzit) im Taunus (Rheinisches Schiefergebirge). $\mathrm{PhD}$ thesis, University of Marburg

Hahn HD, Zankl H (1991) Sedimentation in the Lower Devonian of the Taunus area (Graue Phyllite to Taunusquarzit). Zentralblatt Geol Paläontol Teil I 1990:1509-1520

Hartenfels S (2011) Die globalen Annulata-Events und die DasbergKrise (Famennium, Oberdevon) in Europa und Nord-Afrikahochauflösende Conodonten-Stratigraphie, Karbonat-Mikrofazies, Paläoökologie und Paläodiversität. Münstersche Forsch Geol Paläontol 105:17-527

Hartenfels S, Becker RT (2016) Famennian sedimentation, faunas, and event stratigraphy at Effenberg Quarry (Remscheid-Altena Anticline, Rhenish Massif). Münstersche Forsch Geol Paläontol 108:141-157

Haverkamp J (1991) Detritusanalyse unterdevonischer Sandsteine des Rheinisch-Ardennischen Schiefergebirges und ihre Bedeutung für die Rekonstruktion der sedimentliefernden Hinterlander. Unpubl PhD Thesis, RWTH Aachen, pp 227

Haverkamp J, Hoegen JV, Kramm U, Walter R (1992) Application of $\mathrm{U}-\mathrm{Pb}$-systems from detrital Zircons for palaeogeographic reconstructions a - case-study from the Rhenohercynian. Geodin Acta 5:69-83

Hoffmann N, Jödicke H, Fluche B, Jording A, Müller W (1998) Modellvorstellungen zur Verbreitung potentieller präwestfalischer Erdgas-Muttergesteine in Norddeutschland-Ergebnisse neuer magnetotellurischer Messungen. Z Angew Geol 44:140-158

Huckriede H, Wemmer K, Ahrendt H (2004) Palaeogeography and tectonic structure of allochthonous units in the German part of the Rheno-Hercynian Belt (Central European Variscides). Int J Earth Sci 93:414-431

Jackson SE, Pearson NJ, Griffin WL, Belousova EA (2004) The application of laser ablation-inductively coupled plasma-mass spectrometry to in situ U-Pb zircon geochronology. Chem Geol 211:47-69

Jansen U (2016) Brachiopod faunas, facies and biostratigraphy of the Pridolian to lower Eifelian succession in the Rhenish Massif (Rheinisches Schiefergebirge, Germany). In: Becker RT, Königshof P, Brett CE (eds) Devonian climate, Sea Level and Evolutionary Events. Geological Society, London, Special Publications, vol 423, pp 45-122

Kaiser SI, Aretz M, Becker RT (2015) The global Hangenberg Crisis (Devonian-Carboniferous transition): review of a first-order mass extinction. In: Becker RT, Königshof P, Brett CE (eds) Devonian climate, Sea Level and Evolutionary Events. Geological Society, London, Special Publication, vol 423, pp 387-437

Katzung G, Giese U, Walter R, Von Winterfeld C (1993) The Rügen Caledonides, northeast Germany. Geol Mag 130(5):725-730

Keupp H, Kompa R (1984) Mikrofazielle und sedimentologische Untersuchungen an Devon/Karbon Profilen am Nordrand des rechtsrheinischen Schiefergebirges. In: Paproth E, Streel M (eds) The Devonian-Carboniferous Boundary. Courier Forschungsinstitut Senckenberg, vol 67, pp 139-142

Kirnbauer T (1991) Geologie, petrographie und geochemie der pyroklastika des unteren Ems/ Unter-Devon (Porphyroide) im südlichen Rheinischen Schiefergebirge. Geol Abh Hess 92:1-228

Klein C (2016) Testing modern biostratigraphical methods. Springer Spectrum, Berlin

Königshof P, Becker RT, Hartenfels S (2016) The Rhenish Massif as a part of the European Variscides. Münstersche Forsch Geol Palaeontol 108:1-13

Korn D (1984) Die goniatiten der stockumer imitoceras—kalklinsen. Cour Forsch Senckenberg 67:71-89

Korn D (1991) Three-dimensionally preserved clymeniids from the Hangenberg Black Shale of Drewer (Cephalopoda, Ammonoidea; Devonian-Carboniferous boundary, Rhenish Massif). Neues Jahrbuch für Geologie und Paläontologie, Monatshefte, pp 553-563

Korn D (2004) The mid-Famennian ammonoid succession in the Rhenish Mountains: the "annulata Event" reconsidered. Geol Q 48(3):245-252

Korn D, Clausen C-D, Belka Z, Leuteritz K, Luppold FW, Feist R, Weyer D (1994) Die Devon/Karbon-Grenze bei Drewer (Rheinisches Schiefergebirge). Geol Paläontol Westfal 29: 97-147

Kossmat F (1927) Gliederung des variszischen Gebirge Baues. Abh Sächsischen Geol Landesamtes 1:1-39

Krebs W (1979) Devonian basinal facies. In: House MR, Scrutton CT, Bassett MG (eds) The Devonian System. Special Papers in Palaeontology, vol 23, pp 125-139

Kumpan T, Bábek O, Kalvoda J, Matys Grygar T, Frýda J, Becker RT, Hartenfels S (2015) Petrophysical and geochemical signature of the Hangenberg Events: an integrated stratigraphy of the Devonian-Carboniferous boundary interval in the Northern Rhenish Massif (Avalonia, Germany). Bull Geosci 90:667-694

Langenstrassen F (1983) Neritic sedimentation of the lower and middle Devonian in the Rheinische Schiefergebirge East of the River Rhine. In: Martin H, Eder FW (eds) Intracontinental fold belts. Springer, Berlin, pp 43-76

Linnemann U, Pereira F, Jeffries TE, Drost K, Gerdes A (2008) The Cadomian orogeny and the opening of the Rheic Ocean: the diachrony of geotectonic processes constraints by LA-ICP-MS $\mathrm{U}-\mathrm{Pb}$ zircon dating (Ossa-Morena and Saxo-Thuringian Zones, Iberian and Bohemian Massifs). Tectonophysics 461:21-43

Linnemann U, Hofmann M, Romer RL, Gerdes A (2010) Transitional stages between the Cadomian and Variscan Orogenies: basin development and tectonomagmatic evolution of the southern margin of the Rheic Ocean in the Saxo-Thuringian Zone (North Gondwana shelf). In: Linnemann U, Romer RL (eds) Pre-mesozoic geology of Saxo-Thuringia-from the Cadomian Active Margin to the Variscan Orogeny. Schweizerbart Science Publishers, Stuttgart, pp 59-98

Linnemann U, Herbosch A, Liégeois JP, Pin C, Gärtner A, Hofmann M (2012) The Cambrian to Devonian Odyssey of the Brabant Massif within Avalonia: a review with new zircon ages, geochemistry, Sm-Nd isotopes, stratigraphy and palaeogeography. Earth Sci Rev 112:126-154

Loske WP, Miller H (1987) Heavy mineral analysis on lower Devonian rocks of the Ebbe-Anticline (Rheinisches Schiefergebirge). In: Vogel A, Miller H, Greiling R (eds) The Rhenish Massif: 
Structure, evolution, mineral deposits and present geodynamics. Vieweg Teubner, Wiesbaden, pp 37-50

Ludwig KR (2008) Isoplot 3.70. A geochronological toolkit for microsoft excel. Berkley Geochronology Center Special Publication No. 4

Luppold FW, Clausen CD, Korn D, Stoppel D (1994) Devon/KarbonGrenzprofile im Bereich von Remscheid-Altenaer Sattel, Briloner Sattel und Attendorn-Elsper Doppelmulde (Rheinisches Schiefergebirge). Geol Paläontol Westfal 29: 7-69

McCann T (1999) Middle to Late Devonian basin evolution in the Rügen area, NE Germany. Geol Mijnbouw 78:57-71

McLennan SM (2001) Relationships between the trace element composition of sedimentary rocks and upper continental crust. Geochem Geophys Geosyst 2:1021

Nance RD, Gutiérrez-Alonso G, Keppie JD, Linnemann U, Murphy BJ, Quesada C, Strachan RA, Woodcock N (2010) Evolution of the Rheic Ocean. Gondwana Res 17:194-222

Nesbitt HW, Young GM (1982) Early Proterozoic climates and plate motions inferred from major element chemistry of lutites. Nature 299:715-717

Nesbitt HW, Young GM (1984) Prediction of some weathering trends of plutonic and volcanic rocks based on thermodynamic and kinetic considerations. Geochim Cosmochim Acta 48:1523-1534

Nesbitt HW, Young GM, McLennan SM, Keays RR (1996) Effects of chemical weathering and sorting on the petrogenesis of siliciclastic sediments, with implications for provenance studies. J Geol 104:525-542

Nesbitt HW, Fedo CM, Young GM (1997) Quartz and feldspar stability, steady and non-steady-state weathering, and petrogenesis of siliciclastic sands and muds. J Geol 105:173-191

Neumann-Mahlkau P, Ribbert KH (1998) Die Konglomerate der GivetStufe östlich des Brabanter Massivs. Fortschr Geol Rheinl Westfal 37:393-421

Oliveira JT, Pereira Z, Rosa C, Rosa D, Matos J (2005) Recent advances in the study of the stratigraphy and the magmatism of the Iberian Pyrite Belt, Portugal. J Virtual Explor 19:1441-8142

Oncken O, von Winterfeld C, Dittmar U (1999) Accretion of a rifted passive margin: the late Paleozoic Rhenohercynian fold and thrust belt (Middle European Variscides). Tectonics 18:75-91

Oncken O, Plesch A, Weber K, Ricken W, Schrader S (2000) Passive margin detachment during arc-continent collision (Central European Variscides). In: Franke W, Haak V, Oncken O, Tanner D (eds) Orogenic Processes: Quantification and Modelling in the Variscan Belt. The Geological Society of London, Special Publication, vol 179, pp 199-216

Paproth E (1986) An introduction to a field trip to the Late Devonian outcrops in the northern Rheinisches Schiefergebirge (Federal Republic of Germany). Ann Soc Géol Belg 109:275-284

Paproth E (1991) Devonian palaeogeographic development in Germany and adjacent area. In: Albertsen M (ed) The Deeper Subsoil of the North West German Basin. Sedimentology-Tectonic-Hydrocarbons. DGMK-Report 468: 33-52

Paproth E, Streel M (1982) Devonian — carboniferous transitional beds of the northern "Rheinisches Schiefergebirge". IUGS Working Group on the Devonian/Carboniferous Boundary, Guidebook. Liége, pp 1-63

Paproth E, Dreesen R, Thorez J (1986) Famennian paleogeography and event stratigraphy of northwestern Europe. Ann Soc Géol Belg 109:175-186

Paton C, Woodhead JD, Hellstrom JC, Hergt JM, Greig A, Maas R (2010) Improved laser ablation U-Pb zircon geochronology through robust downhole fractionation correction. Geochem Geophys Geosyst 11:36

Perri F, Critelli S, Cavalcante GM, Dominici R, Sonnino M, De Rosa R (2012) Provenance signatures for the Miocene volcaniclastic succession of the Tufiti di Tusa Formation, southern Apennines, Italy. Geol Mag 149:423-442

Petrus JA, Kamber BS (2012) VizualAge: a novel approach to laser ablation ICP-MS U-Pb geochronology data reduction. Geostand Geoanal Res 36:247-270

Pfeiffer H (1954) Der Bohlen bei Saalfeld/Thür. Geol Beiheft 11:1-105

Piske J, Rasch HJ, Neumann E, Zagora K (1994) Geologischer Bau und Entwicklung des Präperms der Insel Rügen und des angrenzenden Seegebietes. Z Geol Wiss 22:211-226

Press S (1986) Detrital spinels from alpinotype source rocks in middle Devonian sediments of the Rhenish Massif. Geol Rundsch 75(2):333-340

Rey P, Burg JP, Casey M (1997) The Scandinavian Caledonides and their relationship to the Variscan Belt. In: Burg JP, Ford M (eds) Orogeny Through Time. Geological Society, London, Special Publications, vol 121, pp 179-200

Ribbert KH (1982) Die Konglomerate des Schwarzbachtales, ein sedimentologisches Modell. Senckenberg Lethaea 63:345-358

Robbins LJ, Lalonde SV, Saito MA, Planavsky NJ, Mloszewska AM, Pecoits E, Scott C, Dupont C, Kappler A, Konhauser KO (2013) Authigenic iron oxide proxies for marine zinc over geological time and implications for eukaryotic metallome evolution. Geobiology 11:295-306

Sánchez Martínez S, Arenas R, Díaz García F, Martínez Catalán JR, Gómez-Barreiro J, Pearce J (2007) Careón ophiolite, NW Spain: suprasubduction zone setting for the youngest Rheic Ocean floor. Geology 35(1):53-56

Schaltegger U, Schmitt AK, Horstwood MSA (2015) U-Th-Pb zircon geochronology by ID-TIMS, SIMS, and laser ablation ICPMS: Recipes, interpretations, and opportunities. Chem Geol 402:89-110

Schmidt G (1924) Zwei Cephalopodenfaunen an der Devon-Carbongrenze im Sauerland. Jahrbuch der Preußischen Geologischen Landesanstalt 44 (for 1923), pp 98-171

Schulz-Dobrick B, Wedepohl KH (1983) The chemical composition of sedimentary deposits in the Rhenohercynian belt of Central Europe. In: Martin H, Eder FW (eds) Intracontinental fold belt. Springer, Berlin, pp 211-229

Sindern S, Warnsloh J, Martin Trautwein-Bruns U, Chatziliadou M, Becker SY, Kramm U (2008) Geochemical composition of sedimentary rocks and imprint of hydrothermal fluid flow at the Variscan front-an example from the RWTH-1 well (Germany). Z Dtsch Ges Geowiss 159:623-640

Sláma J, Kosler J (2012) Effects of sampling and mineral separation on accuracy of detrital zircon studies. Geochem Geophys Geosyst (G3) 13(5):17

Sláma J, Kosler J, Condon DJ, Crowley JL, Gerdes A, Hanchar JM, Horstwood MSA, Morris GA, Nasdala L, Norberg N, Schaltegger U, Schoene B, Tubrett MN, Whitehouse MJ (2008) Plesovice zircon-a new natural reference material for $\mathrm{U}-\mathrm{Pb}$ and $\mathrm{Hf}$ isotopic microanalysis. Chem Geol 249:1-35

Söderlund U, Jarl LG, Persson PO, Stephens MB, Wahlgren CH (1999) Protolith ages and timing of deformation in the eastern, marginal part of the Sveconorwegian orogen, southwestern Sweden. Precambr Res 94:29-48

Środoń J, Drits VA, McCarty DK, Hsieh JCC, Eberl DD (2001) Quantitative XRD analysis of clay-rich rocks from random preparations. Clays Clay Miner 49:514-528

Stets J, Schäfer A (2002) Depositional environments in the lower devonian siliciclastics of the rhenohercynian Basin (Rheinisches Schiefergebirge, W-Germany) — case studies and a model. Contrib Sediment Geol 22:1-77

Sunkel G (1990) Devonischer submariner Vulkanismus im Ostsauerland (Rheinisches Schiefergebirge): vulkanaufbau, magmenzusammensetzung und alteration. Boch Geol Geotech Arb 34:250 
Tribovillard N, Algeo TJ, Lyons TW, Riboulleau A (2006) Trace metals as paleoredox and paleoproductivity proxies: an update. Chem Geol 232:12-32

Tunheng A, Hirata T (2004) Development of signal smoothing device for precise elemental analysis using laser ablation-ICP-mass spectrometry. J Anal At Spectrom 19:932-934

von Raumer JF, Nesbor HD, Stampfli GM (2017) The north subducting Rheic Ocean during the Devonian: consequences for the Rhenohercynian ore sites. Int J Earth Sci 106:2279-2296

Wagener J (1989) U-Pb-age determinations on detrical zircons of Lower Devonian rocks of the Ardenne and the Rheinisches Schiefergebirge. Ann Soc Géol Belg 112(1):259

Wentworth CK (1922) A scale of grade and class terms for clastic sediments. J Geol 30:377-392

Wiedenbeck M, Alle P, Corfu F, Griffin WL, Meier M, Oberli F, Vonquadt A, Roddick JC, Speigel W (1995) Three natural zircon standards for $\mathrm{U}-\mathrm{Th}-\mathrm{Pb}$, Lu-Hf, trace-element and REE analyses. Geostand Newsl 19:1-23
Winter J (2006) Zirkon-Tephrostratigraphie-stratigraphische Abfolge und Genese der Keratophyr-Horizonte des Sauerlandes (Unterdevon. Rheinisches Schiefergebirge). Z Dtsch Ges Geowiss 157(2):155-202

Winter J (2015) Vulkanismus und Kellwasser-Krise-ZirkonTephrostratigraphie, Identifizierung und Herkunft distaler Fallout-Aschenlagen (Oberdevon, Synklinorium von Dinant, Rheinisches Schiefergebirge, Harz). Z Dtsch Ges Geowiss 166(3):227-251

Zeh A, Gerdes A (2010) Baltica- and Gondwana-derived sediments in the mid-German Crystalline Rise (Central Europe): implications for the closure of the Rheic ocean. Gondwana Res 17:254-263

Zeh A, Will TM (2010) The Mid-German Crystalline Zone. In: Linnemann U, Romer RL (eds) Pre-mesozoic geology of Saxo-Thuringia-from the Cadomian Active Margin to the Variscan Orogeny. Schweizerbart Science Publishers, Stuttgart, pp 195-220 